\title{
Elementary Properties of Nested Hilbert Spaces
}

\author{
Alexander Grossmann * \\ New York University \\ Courant Institute of Mathematical Sciences \\ New York, N. Y.
}

Received June 30, 1965

\begin{abstract}
Detailed derivations are given of the simplest properties of nested Hilbert spaces and of operators between them.
\end{abstract}

\section{Introduction}

A nested Hilbert space is, loosely speaking, the union of a family of Hilbert spaces some of which are suitably embedded into others. There exists a "central" Hilbert space $H_{0}$. The remaining spaces come in pairs $H_{r}, H_{\bar{r}}$ which are in duality with respect to the scalar product in $H_{0}$.

Such objects have a "self-dual" character which makes them relatives of ordinary Hilbert spaces. On the other hand, they are general enough to accomodate various improper functions and operators of quantum mechanics. For example, Wick products of fields (without smearing) are operators - in a sense to be discussed below - in a suitable nested Hilbert space.

1.1 Example: Denote by $I$ the ordered set of real numbers. For every $s \in I$, let $H_{s}$ be the Hilbert space of (classes of) complex-valued measurable functions defined on an euclidean space and such that

$$
\int|f(x)|^{2} \exp (-s|x|) d x<\infty .
$$

For $s \geqq r$, denote by $E_{s r}$ the natural embedding which associates, to every $f_{r} \in H_{r}$, the same function considered as an element of $H_{s}$. Notice that $E_{s r}$ is continuous and injective. The range of $E_{s r}$ is dense in $H_{s}$.

Denote by $H_{I}$ the union of all the spaces $H_{s}$. (Actually, this in an algebraic inductive limit; see below.) Denote by $E_{I S}$ the natural embedding of the Hilbert space $H_{s}$ into the vector space $H_{I}$.

* Present address: Institut des Hautes Etudes Scientifiques, Bures-sur-Yvette (Essonne), France. Physics.

This work was prepared under a Ford Foundation Grant for Mathematical

Commun. math. Phys., Vol. 2 
For typographical convenience, write $\bar{s}$ to denote $-s$. If $f$ and $g$ in $H_{I}$ are such that, for some $s \in I, f$ belongs to $E_{I s} H_{s}$ and $g$ to $E_{I \bar{s}} H_{\bar{s}}$ then a scalar product

$$
\langle f \mid g\rangle=\int f^{*}(x) g(x) d x
$$

is defined. Notice that (1.1) has the form of the scalar product in $H_{0}=L^{(2)}$ but that we do not require both $f$ and $g$ to be in $L^{(2)}$. The exponential increase of - say - $f$ at infinity can be compensated by a corresponding decrease in $g$.

It is useful to classify the functions in $H_{I}$ by associating to every such function $f$ the set $J(f) \leqq I$ of all numbers $r$ such that $f \in E_{I r} H_{r}$. For example, if $f$ is, respectively, $\exp \left(-x^{2}\right), 1 /\left(1+x^{2}\right), x^{5}, \exp (2|x|)$ (in one dimension), then $J(f)=(-\infty \infty)=I$, resp. [0 [)$,(0 \infty),(4 \infty)$.

The adjoint of $E_{s r}$ is the mapping

from $H_{s}$ into $H_{r}$.

$$
\left(E_{s r}\right)_{r s}^{*}: f(x) \rightarrow \exp [(r-s)|x|] f(x)
$$

Notice that the mapping

$$
u_{r \bar{r}}: f(x) \rightarrow \exp (r|x|) f(x)
$$

establishes a unitary correspondence between $H_{r}$ and $H_{\bar{r}}$ and that

$$
\left(E_{s r}\right)_{r s}^{*}=u_{r \bar{r}} E_{\bar{r} \bar{s}} u_{\bar{s} s} \text {. }
$$

1.2 Example: Denote by $H_{0}$ the Hilbert space of (classes of) functions $f(x, y)$ which are square integrable with respect to the Lebesgue measure in the plane. Consider in $H_{0}$ the orthonormal basis $\left\{h_{k}(x) h_{j}(y)\right\}$ where $h_{k}(x)$ is the (properly normalized) product of a Hermite polynomial and of $\exp \left(-\frac{1}{2} x^{2}\right)$.

Let $r=r(k, j)(k, j=0,1,2, \ldots)$ be a double sequence of strictly positive numbers. Denote by $I$ the set of all such sequences, partially ordered in the natural way: $r \leqq s$ means that $r(k, j) \leqq s(k, j)$ for all $k, j$. Given any sequence $r \in I$, consider the sequence $\bar{r}$ defined by $\bar{r}(k, j)$ $=1 / r(k, j)$. Notice that the correspondence $r \leftrightarrow \bar{r}$ is an order-reversing involution analogous to the correspondence $s \leftrightarrow-s$ in Example 1.1.

To every $r \in I$ associate the prehilbert space consisting of all finite linear combinations of the vectors $h_{k}(x) h_{j}(y)$; the scalar product of

and of

$$
f(x, y)=\sum_{k} \sum_{j} c_{k j} h_{k}(x) h_{j}(y)
$$

is the number

$$
g(x, y)=\sum_{k} \sum_{j} d_{k j} h_{k}(x) h_{j}(y)
$$

$$
\sum_{k} \sum_{j} c_{k j}^{*} d_{k j} \frac{1}{r^{2}(k, j)}
$$


where the star denotes complex conjugation. Denote by $H_{r}$ the completion of this prehilbert space.

If $r \leqq s$, then $H_{r} \leqq H_{s}$. One can introduce the operators $E_{s r}$ of embedding as in Example 1.1. Consider the unitary mapping $u_{\bar{r} r}$ from $H_{r}$ into $H_{\bar{r}}$, defined by:

$$
u_{\bar{r} r}\left(h_{k}(x) h_{j}(y)\right)=\frac{1}{r^{2}(k, j)} h_{k}(x) h_{j}(y),
$$

linear extension and closure. It can be verified that (1.4) holds.

Consider again, the vector space $H_{I}=\cup H_{r}$; the union is now over all double sequences of positive numbers. Define $E_{I r}$ and $J(f)$ as in Example 1.1. That is : $r \in J(f)$ means $f \in E_{I r} H_{r}$.

At this point, a legitimate question is why we are complicating matters by considering the full partially ordered set $I$, when any cofinal subset of $I$ (in particular, any totally ordered cofinal subset) would give the same supply of elements.

The answer, is roughly, that the set $J(f)$ gives a useful description of the "regularity" of a vector $f$ in $H_{I}$. A mutilation of $I$ can lead to a decrease of the amount of information contained in $J(f)$.

In order to illustrate this, consider in $H_{I}$ the vectors

and

$$
f(x, y)=\delta(x) \exp \left(-\frac{1}{2} y^{2}\right)
$$

$$
g(x, y)=\exp \left(-\frac{1}{2} x^{2}\right) \delta(y) .
$$

Here $\delta$ is the Dirac $\delta$-function. One should clearly require the scalar product $\langle f \mid g\rangle$ to be defined since

$$
\iint \delta(x) \delta(y) \exp \left(-\frac{1}{2} x^{2}-\frac{1}{2} y^{2}\right) d x d y=1 .
$$

This means, as will be seen below, that we require the existence of at least one $r \in I$ such that $r \in J(f)$ and $\bar{r} \in J(g)$. This condition can easily be satisfied if $I$ is the partially ordered set of all double sequences of positive numbers. Indeed, if $\sum_{k} r^{-1}(k, j)<\infty$ for every $j$, then $r \in J(f)$. If $\sum_{j} r(k, j)<\infty$ for every $k$, then $\bar{r} \in J(g)$. These two conditions are compatible: one can take e. g. $r(k, j)=(k / j)^{2}$. On the other hand, consider e. g. the subset $I^{\prime} \subset I$ consisting of double sequences of the form $r(k, j)$ $=r^{\prime}(k+j)$. It is clear that $I^{\prime}$ is cofinal with $I$; however, the above conditions cannot be satisfied within $I^{\prime}$ and even less within a totally ordered subset of $I^{\prime}$.

In Sec. $3 \mathrm{a}$, a nested Hilbert space is defined as an algebraic inductive limit of a family $H_{r}(r \in I)$ of Hilbert spaces. A certain number of conditions are imposed on the set $I$ and on the family $E_{s r}$ of mappings with respect to which the limit is taken. These restrictions will be now discussed in a qualitative way. 
First the conditions on $I$ (see Sec. 2e): The discussion of Example 1.2 has shown that $I$ should in general be only partially ordered. It can be seen from the same example that it would be unduly restrictive to assume that every $r \in I$ is comparable to $o$.

One has to assume, however, that $I$ is directed to the right, because this insures the existence of the inductive limit. The idea that the spaces $H_{r}$ come in pairs $H_{r}, H_{\vec{r}}$ is expressed by postulating the existence, in $I$, of an order-reversing involution $r \leftrightarrow \bar{r}$. The last requirement is the existence of a $o \in I$ such that $\bar{o}=o$. (This condition could be dropped without dramatic consequences.)

Next: The mappings $E_{s r}(s \geqq r)$ are assumed to be injective, continuous and with dense range. For the sake of completeness, the simplest properties of such mappings are derived in Sec. $2 \mathrm{c}$ and $2 \mathrm{~d}$; a class of examples is exhibited in Sec. $2 \mathrm{~g}$. It is also assumed, of course, that the family $E_{s r}(r \geqq s)$ satisfies the usual conditions required to insure the existence of the inductive limit.

One has now to express precisely the idea that $H_{r}$ and $H_{\bar{r}}$ are in duality with respect to the scalar product in $H_{0}$. This is often done by using one of the two spaces as a space of testing functions and by defining the other as a suitable set of linear functionals. For our purposes, this is not convenient: First of all, it destroys the symmetry between $H_{r}$ and $H_{\bar{r}}$ which is one of our main themes. Secondly, the procedure is not directly applicable if $r$ and $\bar{r}$ are not comparable to $o$. For example, each of the vectors (1.5) and (1.6) could with equal justification be called a testing function and a linear functional.

A suitable way of expressing the duality requirement is given in Section $3 \mathrm{a}$. The condition $\left(\mathrm{NH}_{2}\right)$ of that section requires the nesting $E_{\bar{r} \bar{s}}$ to be "unitarily related" to the adjoint $\left(E_{s r}\right)_{r s}^{*}$ of $E_{s r}$. More precisely, it postulates the existence of a family of unitary operators $u_{r \bar{r}}$ such that (1.4) holds.

While $H_{r}$ is a Hilbert space, its image $E_{I r} H_{r}$ under the natural embedding is just a vector subspace of the vector space $H_{I}$. It is useful to consider the family of all vector subspaces of $H_{I}$ which are of the form

for some $r \in I$.

$$
E_{I r} H_{r}
$$

The condition $\left(N H_{1}\right)$ of Sec. 3 a requires that the intersection of any two vector subspaces of the form (1.7) be again a subspace of the form (1.7). It is trivially satisfied if $I$ is totally ordered. In the general case, it insures the existence of "sufficiently" many Hilbert spaces $H_{r}$.

From $\left(\mathrm{NH}_{1}\right)$ one can deduce [with the help of $\left(\mathrm{NH}_{2}\right)$ ] a dual statement: The linear span of any two subspaces of the form (1.7) is again a subspace of the form (1.7). These and related statements are proved in Section $3 \mathrm{~b}$ by an adaptation of methods of L. ScHWARTz [1]. 
Let $H_{I}$ be a nested Hilbert space. What is a suitable definition of an operator in $H_{I}$ ?

A natural answer would be: An operator in $H_{I}$ is a linear mapping defined on a union of subspaces of the form (1.7) and mapping each of these subspace into a subspace of the same form.

For example, convolution by $\exp (|x|)$ is an operator in the nested Hilbert space of Example 1.1. The differentiation and the multiplication by $\delta(x) \delta(y)$ are operators in the nested Hilbert space of Example 1.2. Notice, incidentally, that the first two of these three operators are defined on all of $H_{I}$ and that the last two are not operators in the "wrong" nested Hilbert space.

The above definition is not quite complete; another natural requirement is that the domain of an operator should not be ,artificially restricted". It is technically convenient to insure simultaneously the validity of all these requirements by defining an operator as an element of a suitable algebraic inductive limit of Banach spaces of bounded operators between Hilbert spaces (Section 4a). It follows from this definition that the domain of a sum of operators may well be larger than the intersection of the domains of the summands.

Every operator in a nested Hilbert space has unique adjoint which is an operator in the same space (Section 4c). The correspondence $A \leftrightarrow A^{*}$ between an operator and its adjoint is antilinear, involutory and bijective; everything is happening as if $A$ were a bounded operator in an ordinary Hilbert space. In the examples above, the first and the third operator is selfadjoint.

Actually the sections $4 \mathrm{a}$ to $4 \mathrm{e}$ do not deal with operators in a nested Hilbert space but, more generally, with operators between nested Hilbert spaces. This generality is needed in the applications. For example, one obtains a theorem on the representation of linear functionals (analogous to the classical Riesz-Fréchet theorem) by letting one of the spaces to be the set of complex numbers (Section 4e). Products of operators are studied in Section $4 \mathrm{~d}$. Again, the domain of a product may be larger than the domains of the factors.

A class of examples of nested Hilbert spaces is studied in Sections 5 a to $5 \mathrm{c}$.

The upshot of all this is a Dirac notation supplemented by the systematic use of the sets $J(h)$ and $J(A)$. This machinery will be applied to concrete problems in later papers; its utility will of course always depend on a judicious choice of the space $H_{I}$.

Some of the results of this paper have been stated without proof in a previous publication [2] which is concerned with a particular nested Hilbert space. The definition given in [2] assumes $I$ to be totally ordered and is thus less general than the one given here. 
In a rigged Hilbert space of GEL'FAND and VILENKIN [3], the set $I$ consists of all the integers, and the mappings $E_{s r}$ are required to satisfy some additional conditions. There is not much overlap between the present paper and [3] because we stress the analogy to Hilbert space and avoid the theory of topological vector spaces.

\section{Preliminaries \\ a) Hilbert spaces}

For the terminology, see e. g. Riesz [4] or Dunford-Schwartz [5].

Several Hilbert spaces will be considered simultaneously and denoted by $H_{r}, H_{s}, \ldots$ Elements of $H_{r}$ are denoted by $f_{r}, g_{r}, \ldots$. A bounded linear operator from $H_{r}$ into $H_{s}$ is denoted e. g. by $A_{s r}$. The adjoint of $A_{s r}$ is the bounded linear operator from $H_{s}$ into $H_{r}$, denoted by $\left(A_{s r}\right)_{r s}^{*}$ and defined by

$$
\left(g_{r},\left(A_{s r}\right)_{r s}^{*} f_{s}\right)=\left(A_{s r} g_{r}, f_{s}\right)
$$

for every $g_{r} \in H_{r}$ and every $f_{s} \in H_{s}$. On the 1.h.s. of (2.1) the scalar product is in $H_{r}$; on the r. h. s. it is in $H_{s}$. There is no need to indicate this by $(,)_{r}$ or $(,)_{s}$ since the subscripts are carried by the vectors and the operators. The scalar product $\left(f_{r}, g_{r}\right)$ is linear in $g_{r}$ and antilinear in $f_{r}$.

Similar conventions are adopted for norms: $\left\|f_{r}\right\|$ is the norm in $H_{r}$, and $\left\|A_{s r}\right\|$ the bound norm of $A_{s r}$ (with respect to the norms in $H_{r}$ and $H_{s}$ ).

\section{b) Polar decomposition}

2.1 Proposition: Let $A_{s r}$ be any bounded operator from $H_{r}$ into $H_{s}$. Denote by $\left[A_{s r}\right]_{r r}$ the operator

$$
\left[A_{s r}\right]_{r r}=\left[\left(A_{s r}\right)_{r s}^{*} A_{s r}\right]^{1 / 2}
$$

(positive square root). Then $A_{s r}$ can be decomposed into a product

$$
A_{s r}=W_{s r}\left[A_{s r}\right]_{r r}
$$

where $W_{s r}$ is an isometric correspondence between the closure of the range of $\left[A_{s r}\right]_{r r}$ and the closure of the range of $A_{s r}$.

Proof: For every $f_{r} \in H_{r}$ the equation (2.2) gives

$$
\left(\left[A_{s r}\right]_{r r} f_{r},\left[A_{s r}\right]_{r r} f_{r}\right)=\left(A_{s r} f_{r}, A_{s r} f_{r}\right)
$$

i. e. $\left\|\left[A_{s r}\right]_{r r} f_{r}\right\|=\left\|A_{s r} f_{r}\right\|$. If $h_{r}=\left[A_{s r}\right]_{r r} f_{r}$ and $g_{s}=A_{s r} f_{r}$, write $g_{s}$ $=W_{s r} h_{r}$ and extend by closure. Then $g_{s}=W_{s r} h_{r}=W_{s r}\left[A_{s r}\right]_{r r} f_{r}$ $=A_{s r} f_{r}$ gives (2.3).

\section{c) Nesting}

2.2 Definition: A linear transformation $E_{s r}$ from a Hilbert space $H_{r}$ into a Hilbert space $H_{s}$ is called a nesting if the three conditions below hold: 
$\left(N s_{1}\right) E_{s r}$ is bounded (and defined on all of $H_{r}$ ).

$\left(N s_{2}\right) E_{s r}$ is injective. (Injective means one-to-one into; bijective one-to-one onto.)

$\left(N s_{3}\right)$ The range of $E_{s r}$ is dense in $H_{s}$.

2.3 Proposition: If $E_{s r}$ is a nesting then its adjoint $\left(E_{s r}\right)_{r s}^{*}$ is also a nesting.

Proof: (a) $\left(E_{s r}\right)_{r s}^{*}$ is injective: Let $\left(E_{s r}\right)_{r s}^{*} f_{s}=0$. Then $\left(g_{r},\left(E_{s r}\right)_{r s}^{*} f_{s}\right)$ $=0$ for every $g_{r} \in H_{r}$. Since the range of $E_{s r}$ is dense, it follows that $f_{s}=0$.

(b) The range of $\left(E_{s r}\right)_{r s}^{*}$ is dense: Let $g_{r}$ be such that $\left(g_{r},\left(E_{s r}\right)_{r s}^{*} f_{s}\right)=0$ for every $f_{s} \in H_{s}$. Then $\left(E_{s r} g_{r}, f_{s}\right)=0$ for every $f_{s} \in H_{s}$ so that $E_{s r} g_{r}=0$. Since $E_{s r}$ is injective, it follows that $g_{r}=0$. This proves the proposition.

2.4 Proposition: Let $E_{s r}$ be a bounded linear transformation from $H_{r}$ into $H_{s}$. Let

$$
E_{s r}=U_{s r}\left[E_{s r}\right]_{r r}
$$

be the polar decomposition of $E_{s r}$. Then $E_{s r}$ is a nesting if and only if $U_{s r}$ is unitary and $\left[E_{s r}\right]_{r r}$ is injective.

Proof: (a) If $E_{s r}$ is a nesting, then $\left[E_{s r}\right]_{r r}$ is injective: Indeed, $\left(\left[E_{s r}\right]_{r r} f_{r},\left[E_{s r}\right]_{r r} f_{r}\right)=\left(E_{s r} f_{r}, E_{s r} f_{r}\right)$ so that $\left[E_{s r}\right]_{r r} f_{r}=0$ gives $E_{s r} f_{r}=0$ i. e. $f_{r}=0$.

(b) If $\left[E_{s r}\right]_{r r}$ is injective, then the range of $\left[E_{s r}\right]_{r r}$ is dense in $H_{r}$ : Indeed, if $\left(g_{r},\left[E_{s r}\right]_{r r} f_{r}\right)=0$ for all $f_{r} \in H_{r}$ then $\left(\left[E_{s r}\right]_{r r} g_{r}, f_{r}\right)=0$ so that $\left[E_{s r}\right]_{r r} g_{r}=0$ i. e. $g_{r}=0$.

(c) If $E_{s r}$ is a nesting, then $U_{s r}$ is unitary : Follows from (b) and from Proposition 2.1.

(d) If $U_{s r}$ is unitary and $\left[E_{s r}\right]_{r r}$ injective, then $E_{s r}$ is a nesting. Direct verification.

2.5 Proposition: If $E_{s r}$ and $E_{k s}$ are nestings then the product $E_{k s} E_{s r}$ is a nesting from $H_{r}$ into $H_{k}$.

Proof: $\left(N s_{1}\right)$ and $\left(N s_{2}\right)$ are immediate. In order to prove $\left(N s_{3}\right)$, let $f_{k} \in H_{k}$ be such that $\left(f_{k}, E_{k s} E_{s r} g_{r}\right)=0$ for all $g_{r} \in H_{r}$. Then $\left(\left(E_{k s}\right)_{s k}^{*} f_{k}\right.$, $\left.E_{s r} g_{r}\right)=0$. So $\left(E_{k s}\right)_{s k}^{*} f_{k}=0$ since $E_{s r} H_{r}$ is dense in $H_{s}$. By proposition 2.3 it follows that $f_{k}=0$. This proves the assertion.

\section{d) Nesting and bilinear functionals}

Let $H_{r}$ and $H_{s}$ be Hilbert spaces. A bilinear functional on $H_{r}$ and $H_{s}$ is a complex-valued function $B\left(f_{r}, g_{s}\right)\left(f_{r} \in H_{r}, g_{s} \in H_{s}\right)$ which is linear in the argument $g_{s}$ and antilinear in the argument $f_{r}$. A bilinear functional $B$ is called nondegenerate if (a) for every $f_{r} \in H_{r}$ there exists at least one $g_{s} \in H_{s}$ such that $B\left(f_{r}, g_{s}\right) \neq 0$ and (b) for every $g_{s} \in H_{s}$ there exists at least one $f_{r} \in H_{r}$ such that $B\left(f_{r}, g_{s}\right) \neq 0$.

A bilinear functional $B$ is said to be bounded if there exists a constant $\gamma>0$ such that $\left|B\left(f_{r}, g_{s}\right)\right| \leqq \gamma\left\|f_{r}\right\|\left\|g_{s}\right\|$ for all $f_{r} \in H_{r}, g_{s} \in H_{s}$. 
2.6 Proposition: If $E_{s r}$ is a nesting from $H_{s}$ into $H_{r}$ then the correspondence

$$
\begin{gathered}
\left\{f_{r}, g_{s}\right\} \rightarrow B\left(f_{r}, g_{s}\right)=\left(f_{r}, E_{r s} g_{s}\right) \\
\left(f_{r} \in H_{r}, g_{s} \in H_{s}\right)
\end{gathered}
$$

is a bounded nondegenerate bilinear functional on $H_{r}$ and $H_{s}$. Conversely, given any bounded nondegenerate bilinear functional $B$ on $H_{r}$ and $H_{s}$, the operator $E_{s r}$ defined by the above equality is a nesting.

The proof is easy and may be omitted, since the proposition will not be used.

e) Ordered sets (see e.g. Bourbaki [6])

By order we mean "partial order" (not all pairs of elements have to be comparable). An order-reversing involution in an ordered set $P$ is a bijective correspondence $r \leftrightarrow \bar{r}$ in $P$ such that $\bar{p} \leqq \bar{r}$ if and only if $p \geqq r$ and that $\overline{\bar{r}}=r$.

If $D \leqq P$ is such that $r \in D, p \leqq r$ entails $p \in D$ then $D$ is called an initial subset of $P$. Same terminology for final subsets.

An ordered set $P$ is directed to the right (left) if any two elements $r$, $q \in P$ have at least one common successor $s \geqq r, q$ (predecessor $p \leqq r, q$ ).

By $I$ or by $I^{\prime}, I^{\prime \prime}, \ldots$ we shall denote throughout this paper a set which satisfies the following conditions:

$\left(\mathrm{I}_{1}\right) I$ is directed to the right.

$\left(I_{2}\right)$ In $I$ there is defined an order-reversing involution $r \leftrightarrow \bar{r}$. (Consequently, $I$ is also directed to the left.)

$\left(\mathrm{I}_{3}\right)$ There exists an element $o \in I$ such that $\bar{o}=0$.

Let $I$ and $I^{\prime}$ be two sets that satisfy the above conditions. In the set $I \times I^{\prime}$ of pairs $\left\{r, r^{\prime}\right\}\left(r \in I, r^{\prime} \in I^{\prime}\right)$ we shall consider two order relations:

$$
\begin{array}{ll}
\left\{r, r^{\prime}\right\} \leqq_{1}\left\{s, s^{\prime}\right\} & \text { iff } \quad r \leqq s \text { and } r^{\prime} \leqq s^{\prime} \\
\left\{r, r^{\prime}\right\} \leqq_{2}\left\{s, s^{\prime}\right\} & \text { iff } \quad r \geqq s \text { and } r^{\prime} \leqq s^{\prime} .
\end{array}
$$

Denote by $\left(I \times I^{\prime}\right)_{1}$ the set $I \times I^{\prime}$ endowed with the order (2.5) and by $\left(I \times I^{\prime}\right)_{2}$ the same set endowed with the order (2.6).

2.7 Proposition: If $I$ and $I^{\prime}$ satisfy the conditions $\left(\mathrm{I}_{1}\right),\left(\mathrm{I}_{2}\right),\left(\mathrm{I}_{3}\right)$ then $\left(I \times I^{\prime}\right)_{1}$ and $\left(I \times I^{\prime}\right)_{2}$ also satisfy the same conditions.

Proof: (a) If $s \geqq r, q$ and $p \leqq r, q$ and $s^{\prime} \geqq r^{\prime}, q^{\prime}$ then $\left\{s, s^{\prime}\right\} \geqq$ $\geqq_{1}\left\{r, r^{\prime}\right\},\left\{s, s^{\prime}\right\} \geqq_{1}\left\{q, q^{\prime}\right\},\left\{p, s^{\prime}\right\} \geqq_{\mathbf{2}}\left\{r, r^{\prime}\right\},\left\{p, s^{\prime}\right\} \geqq_{\mathbf{2}}\left\{q, q^{\prime}\right\}$.

(b) The order-reversing involution is defined, in both $\left(I \times I^{\prime}\right)_{1}$ and $\left(I \times I^{\prime}\right)_{2}$, as

$$
\left\{r, r^{\prime}\right\} \leftrightarrow\left\{\bar{r}, \bar{r}^{\prime}\right\} .
$$

(c) Define $o_{1}=o_{2}=\left\{o, o^{\prime}\right\}$. Then $\bar{o}_{1}=o_{1}$ and $\bar{o}_{2}=o_{2}$. This proves the proposition. 
If $S$ is a subset of $I$, then $\bar{S}$ denotes the set of elements $\bar{r}(r \in S)$.

If $\Delta$ is a subset of $I \times I^{\prime}$ then $\Delta^{*}$ denotes the set of elements $\left\{\bar{r}^{\prime}, \bar{r}\right\}$ $\left(\left\{r, r^{\prime}\right\} \in \Delta\right)$. It is a subset of $I^{\prime} \times I$.

\section{f) Algebraic inductive limit}

It will prove convenient to define inductive limits in a way a little more restrictive than is customary (see e. g. Bourbaki [7]).

Let $P$ be an ordered set directed to the right. For every $p \in P$ let $V_{p}$ be a vector space. For every $p \in P$ and every $q \geqq p$ let $E_{q p}$ be an injective linear mapping from $V_{p}$ into $V_{q}$. Assume:

$\left(\operatorname{Ind}_{1}\right) E_{p p}$ is the identity in $V_{p}$ for every $p \in P$.

$\left(\operatorname{Ind}_{2}\right)$ If $q \geqq r \geqq p$ then $E_{q p}=E_{\alpha r} E_{r p}$.

In the disjoint union $\cup_{p \in P} V_{p}$ define an equivalence relation by writing $f_{p} \sim f_{r}$ (where $f_{p} \in V_{p}, f_{r} \in V_{r}$ ) if and only if there exists a $s \geqq p, r$ such that $E_{s p} f_{p}=E_{s r} f_{r}$. The set of classes has a natural structure of vector space which will be denoted by $V_{P}$ and called the algebraic inductive limit of the family $V_{p}$ with respect to the mappings $E_{q \nu}$ and the set $P$. This is written as

$$
V_{P}=\left[V_{p} ; E_{q p} ; P\right] .
$$

The word "algebraic" is a reminder that topology is not involved.

For every $p \in P$ the natural embedding of $V_{p}$ into $V_{P}$ is denoted by $E_{P_{p}}$. It is easy to show that $E_{P p}$ is linear and injective. Furthermore, if $s \geqq r$ then

$$
E_{P r}=E_{P s} E_{s r} .
$$

If $f \in V_{P}$ and if $f=E_{I p} f_{p}$, then $f_{p}$ is called the representative of $f$ in $V_{p}$. If $f_{p}$ is the representative of $f$ in $V_{p}$, if $f_{s}$ is the representative of $f$ in $V_{s}$ and if $s \geqq p$, then

$$
f_{s}=E_{s p} f_{p}
$$

For every $f \in V_{p}$ denote by $J(f)$ the set of all $r \in P$ such that $f \in E_{I r} V_{r}$ (i. e. such that $f$ has a representative in $V_{r}$ ). This $J(f)$ is a non-empty final subset of $P$. It is easy to verify that

$J(0)=P \quad\left(0\right.$ the zero vector of $\left.V_{P}\right)$.

$J(\lambda f)=J(f) \quad\left(\lambda\right.$ a non zero complex number, $\left.f \in V_{P}\right)$.

$J(f+g) \geqq J(f) \cap J(g)$.

If $s \geqq r$, then

by (2.8).

$$
E_{P s} V_{s} \supseteq E_{P r} V_{r}
$$

\section{g) Nestings between $L^{(2)}$-spaces}

For the terminology on measures, see e. g. HaLmos [8].

2.8 Theorem: Let $\mu$ and $\mu^{\prime}$ be two totally $\sigma$-finite positive measures over a set $X$. Assume that $\mu$ and $\mu^{\prime}$ are equivalent, so that both Radon- 
Nikodym derivatives $d \mu / d \mu^{\prime}$ and $d \mu^{\prime} / d \mu$ exist. Assume furthermore that $d \mu^{\prime} / d \mu$ is essentially bounded (with respect to $\mu$ ). Then

(i) $L^{(2)}(X ; \mu) \leqq L^{(2)}\left(X ; \mu^{\prime}\right)$.

(ii) The natural embedding of $L^{(2)}(X ; \mu)$ into $L^{(2)}\left(X ; \mu^{\prime}\right)$ in a nesting.

Proof: (a) The fact that $d \mu^{\prime} / d \mu$ is essentially bounded gives, for every $f \in L^{(2)}(X ; \mu)$

$$
\int|f(x)|^{2} d \mu^{\prime}=\int|f(x)|^{2} \frac{d \mu^{\prime}}{d \mu} d \mu \leqq \text { const } \int|f(x)|^{2} d \mu
$$

which proves (i) and $\left(N s_{1}\right)$.

(b) If $f=0$ in $L^{(2)}\left(X ; \mu^{\prime}\right)$ then $f(x)=0$ almost everywhere with respect to $\mu^{\prime}$. By the equivalence of $\mu$ and of $\mu^{\prime}, f(x)=0$ almost everywhere with respect to $\mu$; so $f=0$ in $L^{(2)}(X ; \mu)$ which verifies $\left(N s_{2}\right)$.

(c) Every simple function on $X$ that belongs to $L^{(2)}(X ; \mu)$ belongs also to $L^{(2)}\left(X ; \mu^{\prime}\right)$. These functions are dense in $L^{(2)}\left(X ; \mu^{\prime}\right)$ so that $\left(N s_{3}\right)$ holds. This proves the theorem.

Consider in particular the case where $X$ is the set $N$ of positive integers. Write $l^{(2)}(\mu)$ for $L^{(2)}(N ; \mu)$.

Let $\left\{\lambda_{k}\right\}$ and $\left\{\lambda_{k}^{\prime}\right\}(k=1,2, \ldots)$ be two sequences of positive numbers. Define the measures $\mu$ and $\mu^{\prime}$ on $N$ by

$$
\mu_{k}=\left(\lambda_{k}\right)^{-2} \text { and } \mu_{k}^{\prime}=\left(\lambda_{k}^{\prime}\right)^{-2} .
$$

Then $l^{(2)}(\mu)$ and $l^{(2)}\left(\mu^{\prime}\right)$ consist of the sequences $z_{k}$ of complex numbers such that

respectively.

$$
\sum_{k}\left|z_{k}\right|^{2}\left(\lambda_{k}\right)^{-2}<\infty \quad \text { and } \quad \sum_{k}\left|z_{k}\right|^{2}\left(\lambda_{k}^{\prime}\right)^{-2}<\infty
$$

2.9 Proposition: Let $\lambda_{k}$ and $\lambda_{k}^{\prime}$ be two sequences of positive numbers such that $\lambda_{k} / \lambda_{k}^{\prime}$ is bounded. Define the measures $\mu$ and $\mu^{\prime}$ by (2.9). Then the natural embedding of $l^{(2)}(\mu)$ into $l^{(2)}\left(\mu^{\prime}\right)$ is a nesting.

Proof: The assertion is a special case of Theorem 2.8.

\section{Nested Hilbert spaces}

\section{a) Definitions}

Let $I$ be a set satisfying the conditions $\left(I_{1}\right),\left(I_{2}\right),\left(I_{3}\right)$ of Section $2 \mathrm{f}$. For every $r \in I$ let $H_{r}$ be a Hilbert space. For every $r \in I$ and every $s \geqq r$ let $E_{s r}$ be a nesting (Section 2c) of $H_{r}$ into $H_{s}$. Assume that the conditions $\left(\operatorname{Ind}_{1}\right),\left(\operatorname{Ind}_{2}\right)$ of Section $2 \mathrm{f}$ hold so that the algebraic inductive limit

$$
H_{I}=\left[H_{r} ; E_{s r} ; I\right]
$$

is defined. This vector space will be called a nested Hilbert space if the following conditions are satisfied: 
$\left(\mathrm{NH}_{1}\right)$ If $r, q$ are any two elements of $I$ then there exists a $p \leqq r, q$ such that $E_{I p} H_{p}$ is the intersection of $E_{I r} H_{r}$ and of $E_{I q} H_{q}$. That is:

$$
E_{I p} H_{p}=E_{I r} H_{r} \cap E_{I q} H_{q}
$$

Notice that $\left(\mathrm{NH}_{1}\right)$ is trivially satisfied if $I$ is totally ordered. In that case $p$ is the smaller of the elements $r, q$.

$\left(\mathrm{NH}_{2}\right)$ For every $q \in I$ there exists a unitary mapping $u_{\bar{q} q}$ from $H_{q}$ onto $H_{\vec{q}}$ such that

$$
\begin{aligned}
& u_{00}=1 \\
& \left(E_{s r}\right)_{r s}^{*}=u_{r \bar{r}} E_{\bar{r} \bar{s}} u_{\bar{s} s} \\
& (r \in I ; s \geqq r) .
\end{aligned}
$$

Notice that

$$
u_{r \bar{r}} u_{\bar{r} r}=1
$$

for every $r \in I$. [Take $r=s$ in $(3.2 \mathrm{~b})$ and use $\left(\operatorname{Ind}_{1}\right)$ of Section $2 \mathrm{f}$.]

3.1 Proposition: Given the spaces $H_{r}(r \in I)$ and the nestings $E_{s r}(s \geqq r)$, there can exist at most one family $u_{\bar{q} q}$ of unitary operators such that $(3.2 \mathrm{a})$ and $(3.2 \mathrm{~b})$ hold.

Proof: (a) Let $q \leqq o$. Then, by $(3.2 \mathrm{a})$ and $(3.2 \mathrm{~b})$,

$$
\left(E_{0 q}\right)_{\bar{q} 0}^{*}=u_{q \bar{q}} E_{\bar{q} 0} \text {. }
$$

This determines $u_{q \bar{q}}$ on $E_{\bar{q} 0} H_{0}$ which is dense in $H_{\bar{q}}$ by $\left(\mathrm{Ns}_{3}\right)$ of Section 2 . Since $u_{\bar{q} q}$ is unitary, it is thus unambiguously defined.

(b) Let $q$ be arbitrary. Let $p$ be a common predecessor of $q$ and of $o$. [Such a $p$ exists by $\left(\mathrm{I}_{1}\right)$ and $\left(\mathrm{I}_{2}\right)$, Section $2 \mathrm{e}$.] Then $u_{\bar{p} p}$ is determined by (a). Furthermore, by $(3.2 \mathrm{~b})$

$$
\left(E_{q p}\right)_{p q}^{*}=u_{p \bar{p}} E_{\bar{p} \bar{q}} u_{\bar{q} q} .
$$

Since $u_{p \bar{p}} E_{\bar{p} \bar{q}}$ is injective, this determines $u_{\bar{q} q}$. So the proposition is proved.

$$
\text { b) The spaces } H_{(r q)} \text { and } H_{[r q]}
$$

In order to exploit the conditions $\left(\mathrm{NH}_{1}\right)$ and $\left(\mathrm{NH}_{2}\right)$, it is technically convenient to introduce some auxialiary spaces which will be studied in this section. The notations and assumptions of Section $3 \mathrm{a}$ will be used without specific reference.

Let $r, q$ be any two elements of $I$. Denote by $H_{r} \oplus H_{q}$ the Hilbert direct sum of the Hilbert spaces $H_{r}$ and $H_{q}$. Elements of $H_{r} \oplus H_{q}$ are pairs $\left(\begin{array}{l}f_{r} \\ g_{q}\end{array}\right)\left(f_{r} \in H_{r}, g_{q} \in H_{q}\right)$. The scalar product in $H_{r} \oplus H_{q}$ of $\left(\begin{array}{l}f_{r} \\ g_{q}\end{array}\right)$ and of $\left(\begin{array}{l}h_{r} \\ k_{q}\end{array}\right)$ is the number $\left(f_{r}, h_{r}\right)+\left(g_{q}, h_{q}\right)$.

Consider in $H_{r} \oplus H_{q}$ the set $H_{[r q]}$ consisting of pairs $\left(\begin{array}{l}f_{r} \\ g_{q}\end{array}\right)$ having the following property: There exists a $t \in E_{I} H_{r} \cap E_{I q} H_{q}$ such that 
$f_{r}=t_{r}$ and $g_{q}=-t_{q}$. In words $: f_{r}$ is the representative of $t$ in $H_{r}$ and $-g_{q}$ is the representative of $t$ in $H_{q}$.

3.2 Proposition: $H_{[r q]}$ is a closed subspace of $H_{r} \oplus H_{q}$.

Proof: Let $s$ be any common successor of $r$ and of $q$. Let $\left(\begin{array}{c}t_{r}^{(n)} \\ -t_{q}^{(n)}\end{array}\right)$ be a convergent sequence of elements of $H_{[r q]}$. Then the sequences $E_{s r} t_{r}^{(n)}$ and $E_{s q} t_{q}^{(n)}$ converge, since $E_{s r}$ and $E_{s q}$ are continuous, and $E_{s r} \lim t_{r}^{(n)}$ $=E_{s q} \lim t_{q}^{(n)}$ since $E_{s r} t_{r}^{(n)}=E_{s q} t_{q}^{(n)}$ for every $n$. This shows that $\left(\begin{array}{c}\lim t_{t}^{(n)} \\ -\lim t_{q}^{(n)}\end{array}\right)$ belongs to $H_{[r q]}$ and so proves the proposition.

So: The set $H_{[r q]}$, endowed with the scalar product inherited from $H_{r} \oplus H_{q}$, is a Hilbert space.

Denote by $H_{(r q)}$ the orthogonal complement of $H_{[r q]}$ in $H_{r} \oplus H_{q}$. That is: $H_{(r q)}$ consists of pairs $\left(\begin{array}{l}f_{r} \\ g_{q}\end{array}\right)$ such that $\left(f_{r}, t_{r}\right)=\left(g_{q}, t_{q}\right)$ for every $t \in E_{I r} H_{r} \cap E_{I q} H_{q}$. With the scalar product inherited from $H_{r} \oplus H_{q}$, it is a Hilbert space.

Let $z$ be any common successor of $r$ and of $q$. That is: $z \geqq r, q$. Consider the mapping $E_{z(r q)}$ from $H_{(r q)}$ into $H_{z}$, defined by:

$$
\begin{gathered}
E_{z(r q)}\left(\begin{array}{l}
f_{r} \\
g_{q}
\end{array}\right)=E_{z r} f_{r}+E_{z q} g_{q} \\
\left(\left(\begin{array}{l}
f_{r} \\
g_{q}
\end{array}\right) \in H_{(r q)}\right) .
\end{gathered}
$$

3.3 Proposition: $E_{z(r q)}$ is a nesting.

Proof: (a) $E_{z(r q)}$ is clearly continuous.

(b) $E_{z(r q)}$ is injective: If $E_{z r} f_{r}+E_{z q} g_{q}=0$, then $E_{z r} f_{r}=-E_{z q} g_{q}$ shows that $f_{r}$ and $-g_{q}$ are the $r$ and $q$ representatives, respectively, of some $t \in E_{I r} H_{r} \cap E_{I q} H_{q}$. So $\left(\begin{array}{l}f_{r} \\ g_{q}\end{array}\right) \in H_{[r q]}$. This gives $f_{r}=0$ and $g_{q}=0$ since $H_{[r q]}$ is the orthogonal complement of $H_{(r q)}$.

(c) Let $\left(\begin{array}{l}f_{r} \\ g_{a}\end{array}\right)$ be any element of $H_{r} \oplus H_{q}$. Then there exists a $\left(\begin{array}{l}f_{r}^{\prime} \\ g_{q}^{\prime}\end{array}\right) \in H_{(r q)}$ such that $E_{z(r q)}\left(\begin{array}{l}f_{r}^{\prime} \\ g_{q}^{\prime}\end{array}\right)=E_{z r} f_{r}+E_{z q} g_{q}$. Indeed, $\left(\begin{array}{l}f_{r} \\ g_{q}\end{array}\right)$ is the sum of its projections on $H_{(r q)}$ and on $H_{[r q]}$ :

$$
\begin{gathered}
\left(\begin{array}{l}
f_{r} \\
g_{a}
\end{array}\right)=\left(\begin{array}{l}
f_{r}^{\prime} \\
g_{a}^{\prime}
\end{array}\right)+\left(\begin{array}{l}
f_{r}^{\prime \prime} \\
g_{a}^{\prime \prime}
\end{array}\right) \\
\left(\begin{array}{l}
f_{r}^{\prime} \\
g_{a}^{\prime}
\end{array}\right) \in H_{(r q)},\left(\begin{array}{l}
f_{r}^{\prime \prime} \\
g_{a}^{\prime \prime}
\end{array}\right) \in H_{[r a]} .
\end{gathered}
$$

Now $E_{z r} f_{r}^{\prime \prime}+E_{z q} g_{q}^{\prime \prime}=0$ by the definition of $H_{[r q]}$. So $E_{z r} f_{r}+E_{z q} g_{q}$ $=E_{z r} f_{r}^{\prime}+E_{z q} g_{q}^{\prime}$ which verifies (c). The element $\left(\begin{array}{l}f_{r}^{\prime} \\ g_{q}^{\prime}\end{array}\right)$ is unique by $(b)$. 
(d) The range of $E_{z(r q)}$ is dense in $H_{z}$ : Notice that $E_{z r} H_{r}$ is dense in $H_{z}$. If $f_{r}$ is any vector in $H_{r}$, then there exists, by (c), a vector $\left(\begin{array}{l}f_{r}^{\prime} \\ g_{\boldsymbol{q}}^{\prime}\end{array}\right) €$ $\in H_{(r q)}$ such that $E_{z(r q)}\left(\begin{array}{l}f_{r}^{\prime} \\ g_{q}^{\prime}\end{array}\right)=E_{z r} f_{r}$. [Take $g_{q}=0$ in (c).]

This completes the proof of Proposition 3.3.

Let $a \leqq r, q$ be any common predecessor of $r$ and of $q$. Consider a mapping $E_{[r q] a}$ of $H_{a}$ into $H_{[r q]}$, defined by

$$
E_{[r q] a} w_{a}=\left(\begin{array}{c}
E_{r a} w_{a} \\
-E_{q a} w_{a}
\end{array}\right)\left(w_{a} \in H_{a}\right) .
$$

It is clear that $E_{[r q] a}$ is continuous. A simple calculation shows that the adjoint $\left(E_{[r q] a}\right)_{a[r q]}^{*}$ of $E_{[r q] a}$ is given by

$$
\begin{gathered}
\left(E_{[r q] a}\right)_{a[r q]}^{*}\left(\begin{array}{c}
t_{r} \\
-t_{q}
\end{array}\right)=\left(E_{r a}\right)_{a r}^{*} t_{r}+\left(E_{q a}\right)_{a q}^{*} t_{q} \\
\left(\left(\begin{array}{c}
t_{r} \\
-t_{q}
\end{array}\right) \in H_{[r q]}\right) .
\end{gathered}
$$

Let $u_{\bar{r} r}$ and $u_{\bar{q} q}$ be the operators defined by $\left(\mathrm{NH}_{2}\right)$. Consider the operator $\left(\begin{array}{lr}u_{\bar{r} r} & 0 \\ 0-u_{\bar{q} q}\end{array}\right)$ which is clearly a unitary mapping from $H_{r} \oplus H_{q}$ onto $H_{\bar{r}} \oplus H_{\bar{q}}$. The adjoint (and inverse) of $\left(\begin{array}{ll}u_{\bar{r} r} & 0 \\ 0-u_{\bar{q} q}\end{array}\right)$ is $\left(\begin{array}{lr}u_{\bar{r}} & 0 \\ 0-u_{\bar{q} \bar{q}}\end{array}\right)$.

3.4 Proposition: The image of $H_{(r q)}$ under $\left(\begin{array}{cc}u_{\bar{r} r} & 0 \\ 0-u_{\bar{q} \alpha}\end{array}\right)$ is $H_{[\bar{r} \bar{q}]}$. The image of $H_{[r q]}$ under $\left(\begin{array}{cc}u_{\bar{r} r} & 0 \\ 0- & u_{\bar{q} q}\end{array}\right)$ is $H_{(\bar{r} \bar{q})}$.

Proof: It is enough to prove the second assertion since $H_{(r q)}$ is the orthogonal complement of $H_{[r a]}$. Let $\left(\begin{array}{c}t_{r} \\ -t_{q}\end{array}\right)$ be any element of $H_{[r a]}$, and $\left(\begin{array}{c}h_{\bar{r}} \\ -h_{\bar{q}}\end{array}\right)$ any element of $H_{[\bar{r} \bar{q}]}$. Then the scalar product of $\left(\begin{array}{ll}u_{\bar{r} r} & 0 \\ 0 & -u_{\bar{q} q}\end{array}\right)$ $\left(\begin{array}{c}t_{r} \\ -t_{q}\end{array}\right)$ and of $\left(\begin{array}{c}h_{\bar{r}} \\ -h_{\bar{q}}\end{array}\right)$ is $\left(h_{\bar{r}}, u_{\bar{r} r} t_{r}\right)-\left(h_{\bar{q}}, u_{\bar{q} q} t_{q}\right)$.

Let $p \leqq r, q$ be such that $E_{I r} H_{r} \cap E_{I q} H_{q}=E_{I p} H_{p}$ [see $\left.\left(\mathrm{NH}_{1}\right)\right]$. Then $t_{r}=E_{r p} t_{p}$ and $t_{q}=E_{q p} t_{p}$.

So:

$$
\begin{aligned}
& \left(h_{\bar{r}}, u_{\bar{r} r} E_{r p} t_{p}\right)-\left(h_{\bar{q}}, u_{\bar{q} q} E_{q p} t_{p}\right) \\
& =\left(\left(E_{r p}\right)_{p r}^{*} u_{r \bar{r}} h_{\bar{r}}, t_{p}\right)-\left(\left(E_{q p}\right)_{p q}^{*} u_{q \bar{q}} h_{\bar{q}}, t_{p}\right) \\
& =\left(u_{p \bar{p}} E_{\bar{p} \bar{r}} h_{\bar{r}}, t_{p}\right)-\left(u_{p \bar{p}} E_{\bar{p} \bar{q}} h_{\bar{q}}, t_{p}\right) \\
& =\left(u_{p \bar{p}} h_{\bar{p}}, t_{p}\right)-\left(u_{p \bar{p}} h_{\bar{p}}, t_{p}\right)=0 .
\end{aligned}
$$

So the image of $\left(\begin{array}{c}t_{r} \\ -t_{q}\end{array}\right)$ belongs to $H_{(\bar{r} \bar{q})}$.

Conversely, let $\left(\begin{array}{l}f_{\bar{r}} \\ g_{\bar{q}}\end{array}\right)$ belong to $H_{(\bar{r} \bar{q})}$; then $\left(f_{\bar{r}}, h_{\bar{r}}\right)=\left(g_{\bar{q}}, h_{\bar{q}}\right)$ for every $h \in E_{I \bar{r}} H_{\bar{r}} \cap E_{I \bar{q}} H_{\bar{q}}$. So: 
Let $\bar{s} \leqq \bar{r}, \bar{q}$ be such that $E_{I \bar{r}} H_{\bar{r}} \cap E_{I \bar{q}} H_{\bar{q}}=E_{I \bar{s}} H_{\bar{s}}$.

Then $h_{\bar{r}}=E_{\bar{r} \bar{s}} h_{\bar{s}}$ and $h_{\bar{q}}=E_{\bar{q} \bar{s}} h_{\bar{s}}$. So

$$
\begin{gathered}
\left(f_{\bar{r}}, E_{\bar{r} \bar{s}} h_{\bar{s}}\right)=\left(g_{\bar{q}}, E_{\bar{q} \bar{s}} h_{\bar{s}}\right), \quad \text { i. e. } \\
\left(f_{\bar{r}}, u_{\bar{r} r}\left(E_{s r}\right)_{r s}^{*} u_{s \bar{s}} h_{\bar{s}}\right)=\left(g_{\bar{q}}, u_{\bar{q} q}\left(E_{s q}\right)_{q s}^{*} u_{s \bar{s}} h_{\bar{s}}\right)
\end{gathered}
$$

or

$$
\left(E_{s r} u_{r \bar{r}} f_{\bar{r}}, u_{s \bar{s}} h_{\bar{s}}\right)=\left(E_{s q} u_{q \bar{q}} g_{\bar{q}}, u_{s \bar{s}} h_{\bar{s}}\right)
$$

for every $h_{\bar{s}} \in H_{\bar{s}}$. This means that

$$
E_{s r} u_{r \bar{r}} f_{\bar{r}}=E_{s q} u_{q \bar{q}} g_{\bar{q}}
$$

i. e. that $u_{r \bar{r}} f_{\bar{r}}=t_{r}, u_{Q \bar{q}} g_{\bar{q}}=t_{q}$ with $t \in E_{I r} H_{r} \cap E_{I q} H_{q}$. So

which means that

$$
\left(\begin{array}{cc}
u_{r \bar{r}} & 0 \\
0-u_{\bar{q} \bar{q}}
\end{array}\right)\left(\begin{array}{l}
f_{\bar{r}} \\
g_{\bar{q}}
\end{array}\right) \in H_{[r q]}
$$

$$
\left(\begin{array}{l}
f_{\bar{r}} \\
g_{\bar{q}}
\end{array}\right) \in\left(\begin{array}{lr}
u_{\bar{r} r} & 0 \\
0-u_{\bar{q} q}
\end{array}\right) H_{[r q]} .
$$

This completes the proof of Proposition 3.4.

Denote by $u_{(\bar{r} \bar{q})[r q]}$ the restriction of $\left(\begin{array}{cc}u_{\bar{r} r} & 0 \\ 0-u_{\bar{q} q}\end{array}\right)$ to $H_{[r q]}$ and by $u_{[\bar{r} \bar{q}](r q)}$ the restriction of $\left(\begin{array}{lr}u_{\bar{r} r} & 0 \\ 0-u_{\bar{q} q}\end{array}\right)$ to $H_{(r q)}$.

3.5 Proposition: Let $a$ be any common predecessor of $r$ and of $q$, so that $\bar{a}$ is a common successor of $\bar{r}$ and of $\bar{q}$. Then

$$
E_{\bar{a}(\bar{r} \bar{q})}=u_{\bar{a} a}\left(E_{[r q] a}\right)_{a[r q]}^{*} u_{[r q](\bar{r} \bar{q})} .
$$

Proof: Let $\left(\begin{array}{l}f_{\bar{r}} \\ g_{\bar{a}}\end{array}\right)$ be any element of $H_{(\bar{r} \bar{q})}$. Then $E_{\bar{a}(\bar{r} \bar{q})}\left(\begin{array}{l}f_{\bar{r}} \\ g_{\bar{q}}\end{array}\right)=E_{\bar{a} \bar{r}} f_{\bar{r}}+$ $+E_{\bar{a} \bar{q}} g_{\bar{q}}$. On the other hand, by (3.4) and $(3.2 \mathrm{~b})$

$$
\begin{aligned}
& u_{\bar{a} a}\left(E_{[r q] a}\right)_{a[r q]}^{*} u_{[r q](\bar{r} \bar{q})}\left(\begin{array}{l}
f_{\bar{r}} \\
g_{\bar{q}}
\end{array}\right) \\
& =u_{\bar{a} a}\left(E_{[r q] a}\right)_{a[r q]}^{*}\left(\begin{array}{c}
u_{r \bar{r}} f_{\bar{r}} \\
-u_{q \bar{q}} g_{\bar{q}}
\end{array}\right) \\
& =u_{\bar{a} a}\left(\left(E_{r a}\right)_{a r}^{*} u_{r \bar{r}} f_{\bar{r}}+\left(E_{q a}\right)_{a \bar{q}}^{*} u_{q \bar{q}} g_{\bar{q}}\right) \\
& =E_{\bar{a} \bar{r}} f_{\bar{r}}+E_{\bar{a} \bar{q} \bar{q}} g_{\bar{q}}
\end{aligned}
$$

which proves the assertion.

Corollary: $E_{[r q] a}$ is a nesting.

Indeed, this assertion follows from Propositions 2.3 and 2.5.

3.6 Proposition: $E_{[r q] a}$ is bijective if and only if $E_{\bar{a}(\bar{r} \bar{q})}$ is bijective.

Indeed, a nesting is bijective if and only if its adjoint is bijective; this can e.g. be seen from the polar decomposition, Proposition 2.4. So the assertion follows from Eq. (3.5).

3.7 Proposition: Let $z$ be some common successor of $r$ and of $q$. Then $E_{z(r q)}$ is bijective if and only if $E_{I z} H_{z}=E_{I r} H_{r}+E_{I q} H_{q}$, i. e. if $E_{I z} H_{z}$ is the linear span in $H_{I}$ of $E_{I r} H_{r}$ and of $E_{I q} H_{q}$. 
Proof: (a) Assume that $E_{z(r q)}$ is bijective. This means that every $h_{z} \in H_{z}$ can be written as $E_{z r} f_{r}+E_{z q} g_{q}$, where $\left(\begin{array}{l}f_{r} \\ g_{q}\end{array}\right) \in H_{(r q)}$. So $E_{I z} H_{z} \leqq$ $\leqq E_{I r} H_{r}+E_{I q} H_{q}$.

On the other hand, $E_{I z} H_{z} \supseteqq E_{I r} H_{r}$ and $E_{I z} H_{z} \supseteqq E_{I q} H_{q}$ since $z \geqq r$, $q$. So $E_{I z} H_{z} \geqq E_{I r} H_{r}+E_{I q} H_{q}$ since $E_{I z} H_{z}$ is a vector space. Consequently $E_{I z} H_{z}=E_{I r} H_{r}+E_{I q} H_{q}$.

(b) Assume that $E_{I z} H_{z}=E_{I r} H_{r}+E_{I q} H_{q}$.

This means that every $h=E_{I z} h_{z} \in E_{I z} H_{z}$ can be written as a sum $h=f+g, \quad f=E_{I r} f_{r} \in E_{I r} H_{r} \quad$ and $g=E_{I q} g_{q} \in E_{I q} H_{q}$. So $E_{I z} h_{z}$ $=E_{I z} E_{z r} f_{r}+E_{I z} E_{z q} g_{q}$ which means that $h_{z}=E_{z r} f_{r}+E_{z \alpha} g_{q}$. Consider the vector $\left(\begin{array}{l}f_{r} \\ g_{q}\end{array}\right) \in H_{r} \oplus H_{q}$ and the projection $\left(\begin{array}{l}f_{r}^{\prime} \\ g_{q}^{\prime}\end{array}\right)$ of $\left(\begin{array}{l}f_{r} \\ g_{q}\end{array}\right)$ on $H_{(r q)}$. Then $E_{z(r q)}\left(\begin{array}{l}f_{r}^{\prime} \\ g_{Q}^{\prime}\end{array}\right)=E_{z r} f_{r}+E_{z Q} g_{q}=h_{z}$ [see part (c) in the proof of Proposition 3.3]. Consequently $E_{z(r q)}$ is bijective. This proves the proposition.

3.8 Proposition: Let $a$ be some common predecessor of $r$ and of $q$. Then $E_{[r q] a}$ is bijective if and only if $E_{I a} H_{a}=E_{I r} H_{r} \cap E_{I q} H_{q}$.

Proof: (a) Assume that $E_{[r a] a}$ is bijective. Then every $\left(\begin{array}{c}t_{r} \\ -t_{q}\end{array}\right) \in H_{[r q]}$ can be written as $\left(\begin{array}{c}E_{r a} w_{a} \\ -E_{q a} w_{a}\end{array}\right)$ This means: Given any $t \in E_{I r} H_{r} \cap E_{I q} H_{q}$, there exists a $w=E_{I a} w_{a} \in E_{I a} H_{a}$ such that $t_{r}=E_{r a} w_{a}, t_{q}=E_{q a} w_{a}$; consequently $t=E_{I r} E_{r a} w_{a}=E_{I a} w_{a} \in E_{I a} H_{a}$. So $E_{I r} H_{r} \cap E_{I q} H_{q} \leqq$ $\leqq E_{I a} H_{a}$. On the other hand $E_{I r} H_{r} \cap E_{I q} H_{q} \supseteqq E_{I a} H_{a}$, since $a \leqq r, q$. So $E_{I r} H_{r} \cap E_{I q} H_{q}=E_{I a} H_{a}$.

(b) Conversely, let $E_{I a} H_{a}=E_{I r} H_{r} \cap E_{I q} H_{q}$. This means that every $t \in E_{I r} H_{r} \cap E_{I q} H_{q}$ can be written as $t=E_{I a} t_{a}$. Then every $\left(\begin{array}{c}t_{r} \\ -t_{q}\end{array}\right) \in H_{[r q]}$ is of the form $\left(\begin{array}{c}E_{r_{a}} t_{a} \\ -E_{q a} t_{a}\end{array}\right)$ i. e. is in the range of $E_{[r q] a}$. This shows that $E_{[r q] a}$ is bijective and so proves the proposition.

Summarizing, we have

3.9 Theorem: Let $H_{I}$ be a nested Hilbert spaces and let $r, q$ be any two elements of $I$. Let $s \geqq r, q$ be a common successor of $r$ and of $q$. Then the following four conditions are equivalent:

(i) $E_{I s} H_{s}=E_{I r} H_{r}+E_{I q} H_{q}$.

(ii) $E_{I \bar{s}} H_{\bar{s}}=E_{I \bar{r}} H_{\bar{r}} \cap E_{I \bar{q}} H_{\bar{q}}$.

(iii) $E_{s(r q)}$ is bijective.

(iv) $E_{[\bar{r} \bar{q}] \bar{s}}$ is bijective.

\section{c) Scalar product}

If $h$ is any vector in a nested Hilbert space $H_{I}$, then the final subset $J(h) \leqq I$ is directed to the left. Indeed, let $r \in J(h)$ and $q \in J(h)$. By $\left(\mathrm{NH}_{1}\right)$, 
there exists a $p \leqq r, q$ such that $E_{I p} H_{p}=E_{I r} H_{r} \cap E_{I q} H_{q}$. Then $p \in J(h)$ which verifies the assertion.

Denote by $\bar{J}(h)$ the set of all $r \in I$ such that $\bar{r} \in J(h)$. So $\bar{J}(h) \leqq I$ is an initial subset, directed to the right.

3.10 Theorem: Let $f \in H_{I}$ and $g \in H_{I}$ be such that $\bar{J}(f) \cap J(g)$ is not empty. Then the number $\left(u_{r \bar{r}} f_{\bar{r}}, g_{r}\right)=\left(f_{\bar{r}}, u_{\bar{r} r} g_{r}\right)$ is independent of the choice of $r \in \bar{J}(f) \cap J(g)$.

Proof: (a) The intersection $\bar{J}(f) \cap J(g)$ is directed to the right and to the left.

(b) Let $r \in \bar{J}(f) \cap J(g)$ and $q \in \bar{J}(f) \cap J(g)$. Let $p \in \bar{J}(f) \cap J(g)$ and $z \in \bar{J}(f) \cap J(g)$ be such that $p \leqq r, q \leqq z$. Then $\bar{z} \leqq \bar{r}, \bar{q} \leqq \bar{p}$ and consequently, by $(3.2 \mathrm{~b})$,

Similarly

$$
\begin{aligned}
& \left(f_{\bar{r}}, u_{\bar{r} r} g_{r}\right)=\left(E_{\bar{r} \bar{z}} f_{\bar{z}}, u_{\bar{r} r} E_{r \nu} g_{p}\right) \\
& =\left(\left(E_{z r}\right)_{r z}^{*} u_{z \bar{z}} f_{\bar{z}}, E_{r p} g_{p}\right)=\left(u_{z \bar{z}} f_{\bar{z}}, E_{z p} g_{p}\right) \\
& =\left(u_{z \bar{z}} f_{\bar{z}}, g_{z}\right) .
\end{aligned}
$$

$$
\left(f_{\bar{q}}, u_{\bar{q} q} g_{q}\right)=\left(u_{z \bar{z}} f_{\bar{z}}, g_{z}\right),
$$

which proves the theorem.

Notice that the directedness of $\bar{J}(f) \cap J(g)$ was necessary to insure the existence of the representatives $f_{\bar{z}}$ and $g_{p}$.

3.11 Definition: If $f$ and $g$ are vectors in $H_{I}$ such that $\bar{J}(f) \cap J(g)$ is not empty, then the number $\left(u_{r \bar{r}} f_{\bar{r}}, g_{r}\right)(r \in \bar{J}(f) \cap J(g))$ will be called the scalar product of $f$ and of $g$. It will be denoted by $\langle f \mid g\rangle$.

The scalar product is not defined for all pairs of vectors but precisely for pairs such that $\bar{J}(f) \cap J(g)$ is not empty.

If $o \in J(f)$ and $o \in J(g)$ then $\langle f \mid g\rangle=\left(f_{0}, g_{0}\right)$. So the scalar product in $H_{I}$ is an extension of the scalar product in $H_{0}$.

It is now necessary to show that the existence of $\langle f \mid g\rangle$ and of $\langle f \mid h\rangle$ implies the existence of $\langle f \mid g+h\rangle$.

For every $f \in H_{I}$, denote by $H_{\bar{J}(f)}$ the union

$$
H_{\bar{J}(f)}=\bigcup_{r \in \bar{J}(f)} E_{I r} H_{r} .
$$

3.12 Proposition: $H_{\bar{J}(f)}$ is a vector subspace of $H_{I}$.

Proof: Let $g \in H_{\bar{J}(f)}$ and $h \in H_{\tilde{J}(f)}$. This means that there exists a $r \in J(f)$ such that $g \in E_{I \bar{r}} H_{\bar{r}}$, and that there exists a $q \in J(f)$ such that $h \in E_{I \bar{q}} H_{\bar{q}}$. Let $p \leqq r, q$ be such that $E_{I p} H_{p}=E_{I q} H_{q} \cap E_{I r} H_{r}$. Then $p \in J(f)$ and $\bar{p} \in \bar{J}(f)$. Notice that $E_{I \bar{p}} H_{\bar{p}}=E_{I \bar{q}} H_{\bar{q}}+E_{I \bar{r}} H_{\bar{r}}$, by Theorem 3.9. So $h+g \in E_{I \bar{p}} H_{\bar{p}} \leqq H_{\bar{J}(f)}$ which proves the assertion. 
If $\langle f \mid g\rangle$ is defined, then $\langle g \mid f\rangle$ is also defined since $\bar{J}(g) \cap J(f)$ consists of all elements $r$ such that $\bar{r} \in \bar{J}(f) \cap J(g)$. Furthermore $\langle g \mid f\rangle$ is the complex conjugate of $\langle f \mid g\rangle$ since

$$
\left(u_{\bar{r} r} g_{r}, f_{\bar{r}}\right)^{*}=\left(g_{r}, u_{r \bar{r}} f_{\bar{r}}\right)^{*}=\left(u_{r \bar{r}} f_{\bar{r}}, g_{r}\right) .
$$

Let $f, g, h \in H_{I}$ be such that $\langle f \mid g\rangle$ and $\langle f \mid h\rangle$ are defined. Let $\lambda$ and $\mu$ be arbitrary complex numbers. Then $\langle f \mid \lambda g+\mu h\rangle$ is defined and

$$
\langle f \mid \lambda g+\mu h\rangle=\lambda\langle f \mid g\rangle+\mu\langle f \mid h\rangle .
$$

Also, $\langle\lambda g+\mu h \mid f\rangle$ is defined and

$$
\langle\lambda g+\mu h \mid f\rangle=\lambda^{*}\langle g \mid f\rangle+\mu^{*}\langle h \mid f\rangle .
$$

These assertions follow from Proposition 3.12.

3.13 Proposition: Assume that the set $I$ has the following property:

$\left(\mathrm{I}_{4}\right)$ If $s \in I$ is comparable to $\bar{s}$, then $s$ is comparable to $o$.

Let $f \in H_{I}$ be such that $f \neq 0$ and that $\langle f \mid f\rangle$ is defined. Then $\langle f \mid f\rangle>0$.

Proof: The assumption that $\langle f \mid f\rangle$ is defined means that $\bar{J}(f) \cap J(f)$ is not empty. Let $r$ be any element of $\bar{J}(f) \cap J(f)$. Then $\bar{r} \in \bar{J}(f) \cap J(f)$, since $\bar{J}(f) \cap J(f)$ is symmetric with respect to the involution $r \leftrightarrow \bar{r}$. Let $s \in \bar{J}(f) \cap J(f)$ be a common successor of $r$ and of $\bar{r}$. Such an $s$ exists since $\bar{J}(f) \cap J(f)$ is directed to the right. Then $\bar{s}$ is a common predecessor of $r$ and of $\bar{r}$; consequently $\bar{s} \leqq s$. By the assumption $\left(\mathrm{I}_{4}\right)$, then, $s$ is comparable to $o$ and $\bar{s} \leqq o \leqq s$. Since $\bar{J}(f) \cap J(f)$ is the intersection of a final and an initial subset of $I$, it follows that $o \in \bar{J}(f) \cap J(f)$. Consequently $\langle f \mid f\rangle=\left(f_{0}, f_{0}\right)>0$, q. e. d.

\section{Operators}

\section{a) Definitions}

Let $H_{I}=\left[H_{r} ; E_{s r} ; I\right]$ and $F_{I^{\prime}}=\left[F_{r^{\prime}} ; E_{s^{\prime} r^{\prime}} ; I^{\prime}\right]$ be nested Hilbert spaces. Let $\left(I \times I^{\prime}\right)_{2}$ be the ordered set defined by (2.6).

For every pair $\left\{r, r^{\prime}\right\} \in\left(I \times I^{\prime}\right)_{2}$, let $L\left(r ; r^{\prime}\right)$ be the Banach space of all bounded linear operators from $H_{r}$ into $F_{r^{\prime}}$. If $\left\{s, s^{\prime}\right\} \geqq_{\mathbf{2}}\left\{r, r^{\prime}\right\}$ then the correspondence

$$
E_{2}\left(s, s^{\prime} ; r, r^{\prime}\right): A_{r^{\prime} r} \rightarrow E_{s^{\prime} r^{\prime}} A_{r^{\prime} r} E_{r s}
$$

is an injective linear mapping from $L\left(r ; r^{\prime}\right)$ into $L\left(s ; s^{\prime}\right)$.

In order to verify that $E_{2}\left(s, s^{\prime} ; r, r^{\prime}\right)$ is injective, let $E_{s^{\prime} r^{\prime}} A_{r^{\prime} r} E_{r s}=0$. This means $E_{s^{\prime} r^{\prime}} A_{r^{\prime} r} E_{r s} f_{s}=0$ for every $f_{s} \in H_{s}$. Since $E_{s^{\prime} r^{\prime}}$ is injective, it follows that $A_{r^{\prime} r} E_{r s} f_{s}=0$ for every $f_{s} \in H_{s}$. So $E_{r s} H_{s}$ has to be contained in the null-space of $A_{r^{\prime} r}$ which is a closed subspace of $H_{r}$ since $A_{r^{\prime} r}$ is continuous. Now $E_{r s} H_{s}$ is dense in $H_{r}$ so that the null-space of $A_{r^{\prime} r}$ is all of $H_{r}$. This means $A_{r^{\prime} r}=0$.

The family $E_{\mathbf{2}}\left(s, s^{\prime} ; r, r^{\prime}\right)\left(\left\{r, r^{\prime}\right\} \in\left(I \times I^{\prime}\right)_{2},\left\{s, s^{\prime}\right\} \geqq_{\mathbf{2}}\left\{r, r^{\prime}\right\}\right)$ satisfies the conditions $\left(\operatorname{Ind}_{1}\right),\left(\operatorname{Ind}_{2}\right)$ of Section $2 f$.

Commun. math. Phys., Vol. 2 
4.1 Definition: Denote by $L\left(H_{I} ; F_{I^{\prime}}\right)$ the algebraic inductive limit of the vector spaces $L\left(r ; r^{\prime}\right)$ with respect to the mappings $E_{2}\left(s, s^{\prime} ; r, r^{\prime}\right)$.

An element of $L\left(H_{I} ; F_{I^{\prime}}\right)$ will be called an operator from $H_{I}$ into $F_{I^{\prime}}$. This name will be justified in Section $4 \mathrm{~b}$.

4.2 Notation: The natural embedding of $L\left(r ; r^{\prime}\right)$ into $L\left(H_{I} ; F_{I^{\prime}}\right)$ will be denoted by $E_{2}\left(I \times I^{\prime} ; r, r^{\prime}\right)$.

The representative of $A \in L\left(H_{I} ; F_{I^{\prime}}\right)$ in $L\left(r, r^{\prime}\right)$ will be denoted by $A_{r^{\prime} r}$.

The set of pairs $\left\{r, r^{\prime}\right\}$ such that $A$ has a representative in $L\left(r, r^{\prime}\right)$ is denoted by $J(A)$ in accordance with the conventions of Section $2 \mathrm{f}$. It is a final subset of $\left(I \times I^{\prime}\right)_{2}$.

It is convenient to introduce the sets

$$
\begin{aligned}
& D(A)=p r_{I} J(A), \\
& R(A)=p r_{I^{\prime}} J(A) .
\end{aligned}
$$

That is: $D(A)$ is the initial subset of $I$ consisting of elements $r$ such that $\left\{r, r^{\prime}\right\} \in J(A)$ for at least one $r^{\prime} \in I^{\prime}$.

$R(A)$ is the final subset of $I^{\prime}$ consisting of elements $r^{\prime}$ such that $\left\{r, r^{\prime}\right\} \in J(A)$ for at least one $r \in I$.

\section{b) The vector $A h$}

4.3 Proposition: Let $A \in L\left(H_{I} ; F_{I^{\prime}}\right)$ and let $h \in H_{I}$ be such that

$$
D(A) \cap J(h) \neq \phi .
$$

[i. e. that the intersection of the initial subset $D(A) \leqq I$ and of the final subset $J(h) \leqq I$ is not empty.] Then the vector $E_{I^{\prime} r^{\prime}} A_{r^{\prime} r} h_{r}$ is independent of the choice of $r \in D(A) \cap J(h)$ and of the choice of $\left\{r, r^{\prime}\right\} \in J(A)$.

Proof: Let $\left\{r, r^{\prime}\right\} \in J(A),\left\{q, q^{\prime}\right\} \in J(A), r \in J(h)$ and $q \in J(h)$. Since $J(h)$ is directed to the left there exists a common predecessor $p$ of $r$ and of $q$ in $J(h)$. Let $s^{\prime}$ be any common successor of $r^{\prime}$ and of $q^{\prime}$. Then $\left\{p, s^{\prime}\right\} \in J(A)$ and $A_{s^{\prime} p}=E_{s^{\prime} r^{\prime}} A_{r^{\prime} r} E_{r p}=E_{s^{\prime} q^{\prime}} A_{q^{\prime} q} E_{q p^{\prime}}$.

Consequently $E_{s^{\prime} r} A_{r^{\prime} r} h_{r}=E_{s^{\prime} r^{\prime}} A_{r^{\prime} r} E_{r p} h_{p}=A_{s^{\prime} p} h_{p}=E_{s^{\prime} q^{\prime}} A_{q^{\prime} q^{\prime}} h_{q}$ which proves the assertion.

The vector $E_{I^{\prime} r^{\prime}} A_{r^{\prime} r} h_{r}$ will be denoted by $A h$ and called the image of $h$ under $A$.

So: $A h$ is defined if and only if $D(A) \cap J(h)$ is not empty.

We have to verify now that the set $J(A)$ has properties analogous to those established in Section $3 \mathrm{c}$.

Let $r, q \in I$ and $r^{\prime}, q^{\prime} \in I^{\prime}$ be such that all four pairs $\left\{r, r^{\prime}\right\},\left\{r, q^{\prime}\right\}$, $\left\{q, r^{\prime}\right\}$ and $\left\{q, q^{\prime}\right\}$ belong to $J(A)$. Consider the bounded linear mapping

$$
\left(\begin{array}{lr}
A_{r^{\prime r}} & A_{r^{\prime} q} \\
-A_{q^{\prime} r} & -A_{q^{\prime} q}
\end{array}\right)
$$


from $H_{r} \oplus H_{q}$ into $F_{r^{\prime}} \oplus F_{q^{\prime}}$. The image of $\left(\begin{array}{l}f_{r} \\ g_{q}\end{array}\right) \in H_{r} \oplus H_{q}$ is

$$
\left(\begin{array}{c}
A_{r^{\prime} r} f_{r}+A_{r^{\prime} q} g_{q} \\
-A_{q^{\prime} r} f_{r}-A_{q^{\prime} q} g_{q}
\end{array}\right)=\left(\begin{array}{c}
(A f)_{r^{\prime}}+(A g)_{r^{\prime}} \\
-(A f)_{q^{\prime}}-(A g)_{q^{\prime}}
\end{array}\right)
$$

where $f=E_{I r} f_{r}$ and $g=E_{I q} g_{q}$. Notice that the range of (4.5) is contained in $F_{\left[r^{\prime} q^{\prime}\right]}$ and that the image of any vector in $H_{[r q]}$ is zero; indeed, if $\left(\begin{array}{l}f_{r} \\ g_{a}\end{array}\right) \in H_{[r a]}$, then $f=-g$. So: The image, under (4.5), of any vector $\left(\begin{array}{l}f_{r} \\ g_{q}\end{array}\right) \in H_{r} \oplus H_{q}$ is the image of the orthogonal projection of $\left(\begin{array}{l}f_{r} \\ g_{q}\end{array}\right)$ on $H_{(r q)}$.

Denote by $A_{\left[r^{\prime} q^{\prime}\right](r q)}$ the restriction of (4.5) to $H_{(r q)}$.

Let $s \geqq r, q$ be such that $E_{I s} H_{s}=E_{I r} H_{r}+E_{I q} H_{q}$. Let $p^{\prime} \leqq r^{\prime}, q^{\prime}$ be such that $E_{1^{\prime} p^{\prime}} F_{p^{\prime}}=E_{I^{\prime} r^{\prime}} F_{r^{\prime}} \cap E_{I q^{\prime}} F_{q^{\prime}}$. Then the bounded mappings $E_{s(r q)}$ and $E_{\left[r^{\prime} q^{\prime}\right] p^{\prime}}$ are bijective by Theorem 3.9. Consequently they have bounded inverses (see e.g. [5], p. 57) which will be denoted by $\left(E_{s(r q)}\right)_{(r q) s}^{-1}$ and $\left(E_{p^{\prime}\left[r^{\prime} q^{\prime}\right]}\right)_{\left[r^{\prime} q^{\prime}\right] p^{\prime}}^{-1}$.

We shall now verify that

$$
E_{r^{\prime} p^{\prime}}\left(E_{\left[r^{\prime} q^{\prime}\right] p^{\prime}}\right)_{p^{\prime}\left[r^{\prime} q^{\prime}\right]}^{-1} A_{\left[r^{\prime} q^{\prime}\right](r q)}\left(E_{s(r q)}\right)_{(r q) s}^{-1} E_{s r}=A_{r^{\prime} r}
$$

and that

$$
E_{q^{\prime} p^{\prime}}\left(E_{\left[r^{\prime} q^{\prime}\right] p^{\prime}}\right)_{p^{\prime}\left[r^{\prime} q^{\prime}\right]}^{-1} A_{\left[r^{\prime} q^{\prime}\right](r q)}\left(E_{s(r q)}\right)_{(r q) s}^{-1} E_{s q}=A_{q^{\prime} q} .
$$

Let $h_{r}$ be any vector in $H_{r}$. Then $\left(E_{s(r q)}\right)_{(r q) s}^{-1} E_{s r} h_{r}$ is the pair $\left(\begin{array}{l}f_{r} \\ g_{q}\end{array}\right) \epsilon$ $\in H_{(r q)}$ such that $E_{s r} f_{r}+E_{s q} g_{q}=E_{s r} h_{r}$. Notice that $E_{I r} f_{r}+E_{I q} g_{q}$ $=E_{I r} h_{r}$. Consequently

Then

$$
A_{\left[r^{\prime} q^{\prime}\right](r q)}\left(\begin{array}{l}
f_{r} \\
g_{q}
\end{array}\right)=\left(\begin{array}{c}
(A f)_{r^{\prime}}+(A g)_{r^{\prime}} \\
-(A f)_{q^{\prime}}-(A g)_{q^{\prime}}
\end{array}\right)=\left(\begin{array}{c}
(A h)_{r^{\prime}} \\
-(A h)_{q^{\prime}}
\end{array}\right)
$$

$$
\left(E_{\left[r^{\prime} q^{\prime}\right] p^{\prime}}\right)_{p^{\prime}\left[r^{\prime} q^{\prime}\right]}^{-1}\left(\begin{array}{c}
(A h)_{r^{\prime}} \\
-(A h)_{q^{\prime}}
\end{array}\right)=(A h)_{p^{\prime}},
$$

and finally $E_{r^{\prime} p^{\prime}}(A h)_{p^{\prime}}=(A h)_{r^{\prime}}=A_{r^{\prime} r} h_{r}$. This proves (4.7a). The proof of $(4.7 \mathrm{~b})$ is the same.

So we have established

4.4 Proposition: Let $A \in L\left(H_{I} ; F_{I^{\prime}}\right)$. Let $r, q \in I$ and $r^{\prime}, q^{\prime} \in I^{\prime}$ be such that all four pairs $\left\{r, r^{\prime}\right\},\left\{r, q^{\prime}\right\},\left\{q, r^{\prime}\right\},\left\{q, q^{\prime}\right\}$ belong to $J(A)$. Let $s \geqq r, q$ be such that $E_{I s} H_{s}=E_{I r} H_{r}+E_{I q} H_{q}$ and $p^{\prime} \leqq r^{\prime}, q^{\prime}$ such that $E_{I^{\prime} p^{\prime}} F_{p^{\prime}}=E_{I^{\prime} r^{\prime}} F_{r^{\prime}} \cap E_{I^{\prime} q^{\prime}} F_{q^{\prime}}$. Then $\left\{s, p^{\prime}\right\} \in J(A)$. The representative of $A$ between $H_{s}$ and $F_{s^{\prime}}$ is

$$
A_{p^{\prime} s}=\left(E_{\left[r^{\prime} q^{\prime}\right] p^{\prime}}\right)_{p^{\prime}\left[r^{\prime} q^{\prime}\right]}^{-1} A_{\left[r^{\prime} q^{\prime}\right](r q)}\left(E_{s(r q)}\right)_{(r q) s}^{-1} .
$$

Here $A_{\left[r^{\prime} q^{\prime}\right](r q)}$ is the restriction of (4.5) to $H_{(r q)}$ and the mappings $E_{s(r q)}$ and $E_{\left[r^{\prime} q^{\prime}\right] p^{\prime}}$ are described in Theorem 3.9. 
4.5 Corollary: For every $A \in L\left(H_{I} ; F_{I^{\prime}}\right)$ the set $D(A)$ has the following property: If $r \in D(A), q \in D(A)$ and if $s \geqq r, q$ is such that $E_{I s} H_{s}$ $=E_{I r} H_{r}+E_{I q} H_{q}$, then $s \in D(A)$. In particular, $D(A)$ is directed to the right.

4.6 Proposition: If $A h$ and $A g$ are defined then $A(h+g)$ is defined. Indeed, let $r \in D(A) \cap J(h), q \in D(A) \cap J(g)$. Let $s \geqq r, q$ be such that $E_{I s} H_{s}=E_{I r} H_{r}+E_{I q} H_{q}$. Then $s \in D(A)$. On the other hand, $s \in J(h) \cap J(g)$ since $J(h)$ and $J(g)$ are final subsets. So $s \in J(h+g) \supseteqq$ $\supseteq J(h) \cap J(g)$. This proves that $D(A) \cap J(h+g)$ is not empty.

In other words: The domain

$$
H_{D(A)}=\bigcup_{r \in D(A)} E_{I r} H_{r}
$$

of $A$ is a vector subspace of $H_{I}$.

4.7 Proposition: If $A \in L\left(H_{I} ; F_{I^{\prime}}\right), B \in L\left(H_{I} ; F_{I^{\prime}}\right)$ and $h \in H_{I}$ are such that $A h$ and $B h$ are defined, then $(A+B) h$ is defined.

Indeed, let $r \in D(A) \cap J(h)$ and $q \in D(B) \cap J(h)$. Let $p \leqq r, q$ be such that $E_{I p} H_{p}=E_{I r} H_{r} \cap E_{I q} H_{q}$. Then $p \in J(h)$. On the other hand, $p \in D(A) \cap D(B)$ since $D(A)$ and $D(B)$ are initial subsets of $I$. So $p \in D(A+B) \geqq D(A) \cap D(B)$. Consequently the intersection $J(h) \cap$ $\cap D(A+B)$ is not empty, q. e. d. It is clear that $(\zeta A) h=A(\zeta h)$ $=\zeta(A h)$ for every complex $\zeta$. So the results of this section can be summarized in the statement that $A h$ is linear in $A$ and in $h$.

\section{c) Adjoint operators and matrix elements}

Let $A \in L\left(H_{I} ; F_{I^{\prime}}\right)$ and let $\left\{r, r^{\prime}\right\} \in J(A)$. Consider the bounded operator $\left(A^{*}\right)_{\bar{r} \bar{r}^{\prime}}$ from $F_{\bar{r}^{\prime}}$ into $H_{\bar{r}}$ defined by

$$
\left(A^{*}\right)_{\bar{r} \bar{r}^{\prime}}=u_{\bar{r} r}\left(A_{r^{\prime} r}\right)_{r r^{\prime}}^{*} u_{r^{\prime} \bar{r}^{\prime}} .
$$

Denote by $E_{2}\left(I^{\prime} \times I ; \bar{r}^{\prime}, \bar{r}\right)$ the natural embedding of $L\left(\bar{r}^{\prime}, \bar{r}\right)$ into $L\left(F_{I^{\prime}} ; H_{I}\right)$ (see Section $4 \mathrm{a}$. The roles of $I$ and $I^{\prime}$ are here interchanged).

4.8 Proposition: The operator $A^{*}=E_{2}\left(I^{\prime} \times I ; \bar{r}^{\prime}, \bar{r}\right)\left(A^{*}\right)_{\bar{r} \bar{r}^{\prime}}$ is independent of the choice of $\left\{r, r^{\prime}\right\} \in J(A)$; so the notation on the l. h. s. of $(4.10)$ is justified.

Proof: Let $\left\{r, r^{\prime}\right\} \in J(A)$ and $\left\{q, q^{\prime}\right\} \in J(A)$. Let $\left\{s, s^{\prime}\right\}$ be any common successor, in $\left(I \times I^{\prime}\right)_{2}$, of $\left\{r, r^{\prime}\right\}$ and of $\left\{q, q^{\prime}\right\}$. Then $A_{s^{\prime} s}=E_{s^{\prime} r^{\prime}} A_{r^{\prime} r} E_{r s}$ $=E_{s^{\prime} q^{\prime}} A_{q^{\prime} q} E_{q s^{\prime}}$. The adjoint of the last equality becomes, with the help of $(3.2 \mathrm{~b})$ and $(4.10)$,

$$
E_{\bar{s} \bar{r}}\left(A^{*}\right)_{\bar{r} \bar{r}^{\prime}} E_{\bar{r}^{\prime} \bar{s}^{\prime}}=E_{\overline{\bar{s}} \bar{q}}\left(A^{*}\right)_{\bar{q} \bar{q}^{\prime}} E_{\bar{q}^{\prime} \bar{s}^{\prime}} \cdot
$$

This proves the proposition. 
The operator $A^{*} \in L\left(F_{I^{\prime}} ; H_{I}\right)$ defined by Proposition 4.4 is called the adjoint of the operator $A \in L\left(H_{I} ; F_{I^{\prime}}\right)$.

The proof of Proposition 4.4 shows also that $J\left(A^{*}\right) \supseteqq J^{*}(A)$ where $J^{*}(A)$ is defined as in Section $2 \mathrm{e}$.

The definition (4.10) gives immediately that

$$
A^{* *}=A
$$

for every $A \in L\left(H_{I} ; F_{I^{\prime}}\right)$. [It is enough to verify the equality (4.11) for one representative since an operator is determined by any one of its representatives.] It follows that $J\left(A^{* *}\right)=J(A) \geqq J^{*}\left(A^{*}\right)$. So $J^{*}(A) \geqq$ $\supseteq J\left(A^{*}\right)$ and finally

$$
J^{*}(A)=J\left(A^{*}\right) .
$$

In particular, taking the $I$-projections and $I^{\prime}$-projections we obtain

and

$$
D\left(A^{*}\right)=\bar{R}(A)
$$

$$
R\left(A^{*}\right)=\bar{D}(A) \text {. }
$$

4.9 Theorem: The correspondence $A \leftrightarrow A^{*}$ between $L\left(H_{I} ; F_{I^{\prime}}\right)$ and $L\left(F_{I^{\prime}} ; H_{I}\right)$ is antilinear, involutory and bijective.

Proof: (a) The antilinearity follows from the antilinearity of the correspondence (4.10) between $A_{r^{\prime} r}$ and $\left(A^{*}\right)_{\bar{r} \bar{r}^{\prime}}$, and from the linearity of $E_{2}\left(I^{\prime} \times I ; \bar{r}^{\prime}, \bar{r}\right)$.

(b) The correspondence $A \leftrightarrow A^{*}$ is involutory by (4.11).

(c) The correspondence $A \rightarrow A^{*}$ is injective: If $\left(A^{*}\right)_{\bar{r} \bar{r}^{\prime}}=0$, then $A_{r^{\prime} r}=0$ since the operators $u_{\bar{r} r}$ and $u_{r^{\prime} \bar{r}^{\prime}}$ are unitary. The correspondence $A \rightarrow A^{*}$ is bijective since every $B \in L\left(F_{I}, H_{I} ;\right)$ can be written as $B=A^{*}$ with $A=B^{*}$. This completes the proof of the theorem.

If $h \in H_{I}$ and $f \in F_{I^{\prime}}$, then $J(h) \times \bar{J}(f)$ denotes the set of all pairs $\left\{r, r^{\prime}\right\}$ such that $r \in J(h)$ and $\bar{r}^{\prime} \in J(f)$. It is a non-empty initial subset of $\left(I \times I^{\prime}\right)_{2}$.

Let $A \in L\left(H_{I} ; F_{I^{\prime}}\right), h \in H_{I}$ and $f \in F_{I^{\prime}}$ be such that the intersection of $J(A)$ and of $J(h) \times \bar{J}(f)$ is not empty. This means that there exists a $\left\{r, r^{\prime}\right\} \in I \times I^{\prime}$ such that $r \in J(h), r^{\prime} \in \bar{J}(f)$ and $\left\{r, r^{\prime}\right\} \in J(A)$. Then $r \in J(h) \cap D(A)$ so that $A h$ is defined. Furthermore $A h=E_{I^{\prime} r^{\prime}} A_{r^{\prime} r} h_{r}$ so that $r^{\prime} \in J(A h)$. Consequently $\bar{J}(f) \cap J(A h)$ is not empty so that the scalar product $\langle f \mid A g\rangle$ is defined (in $F_{I^{\prime}}$ ). Denote this scalar product by $\langle f|A| h\rangle$ and call it the matrix element of $A$ between $h$ and $f$.

So: The matrix element $\langle f|A| h\rangle$ is defined if and only if the initial subset $J(h) \times \bar{J}(f) \leqq\left(I \times I^{\prime}\right)_{2}$ and the final subset $J(A) \leqq\left(I \times I^{\prime}\right)_{2}$ have a non-empty intersection.

It is important to notice that there can exist pairs of vectors $h \in H_{I}$, $f \in F_{I}$, such that $\langle f \mid A h\rangle$ is defined and that $\langle f|A| h\rangle$ is not defined. For 
example, let $A$ and $h$ be such that $A h$ is defined and that $A h=0$. Then $\langle f \mid A h\rangle$ is defined for all $f \in F_{I^{\prime}}$, since $J(A h)=J(0)=I^{\prime}$. On the other hand, the matrix element $\langle f|A| h\rangle$ need not be defined for all $f \in F_{I^{\prime}}$ since the intersection of $J(h) \times \bar{J}(f)$ and of $J(A)$ may well be empty.

In general: If $\left\{r, r^{\prime}\right\} \in J(A)$ then $r^{\prime} \in J(A h)$ for all $h \in E_{I r} H_{r}$. This ensures a common "goodness" of all vectors in $A E_{I r} H_{r}$. Individual vectors $A h\left(h \in E_{I r} H_{r}\right)$ may however have much larger $J(A h)$ than this assured minimum. So $J\left(h^{\prime}\right) \geqq J(h)$ does not imply that $J\left(A h^{\prime}\right) \geqq J(A h)$. Consequently, the existence of $\langle f \mid A h\rangle$ does not imply the existence of $\left\langle f \mid A h^{\prime}\right\rangle$ for all $h^{\prime}$ such that $J\left(h^{\prime}\right) \geqq J(h)$. On the other hand, the existence of $\langle f|A| h\rangle$ [i. e. the condition $(J(h) \times \bar{J}(f)) \cap J(A) \neq \phi$ ], does imply the existence of $\left\langle f^{\prime}\left|A^{\prime}\right| h^{\prime}\right\rangle$ for all $f^{\prime}, A^{\prime}, h^{\prime}$ such that $J\left(f^{\prime}\right) \geqq J(f)$, $J\left(A^{\prime}\right) \geqq J(A)$ and $J\left(h^{\prime}\right) \geqq J(h)$.

4.10 Proposition: If $\langle f|A| h\rangle$ is defined, then $A^{*} f$ and $\left\langle A^{*} f \mid h\right\rangle$ (scalar product in $H_{I}$ ) are defined. Furthermore $\left\langle A^{*} f \mid h\right\rangle=\langle f|A| h\rangle$ $=\langle f \mid A h\rangle$.

Proof: Let $\left\{r, r^{\prime}\right\} \in J(A), \quad r \in J(h), r^{\prime} \in J(f)$. Then $\left\{\bar{r}^{\prime}, \bar{r}\right\} \in J^{*}(A)$ $=J\left(A^{*}\right)$. So $\bar{r}^{\prime} \in D\left(A^{*}\right) \cap J(f)$ which shows that $A^{*} f$ is defined. Furthermore $\bar{r} \in J\left(A^{*} f\right)$ which shows that $\bar{J}\left(A^{*} f\right) \cap J(h)$ is not empty so that $\left\langle A^{*} f \mid h\right\rangle$ is defined. Finally, by (4.10)

$$
\begin{aligned}
\left\langle A^{*} f \mid h\right\rangle & =\left(u_{r \bar{r}}\left(A^{*} f\right)_{\bar{r}}, h_{r}\right) \\
& =\left(u_{r \bar{r}} u_{\bar{r} r}\left(A_{r^{\prime} r}\right)_{r r^{\prime}}^{*} u_{r^{\prime} \bar{r}^{\prime}} f_{\bar{r}^{\prime}}, h_{r}\right) \\
& =\left(u_{r^{\prime} \bar{r}^{\prime}} f_{\bar{r}^{\prime}}, A_{r^{\prime} r} h_{r}\right)=\langle f \mid A h\rangle
\end{aligned}
$$

which proves the assertion.

4.11 Proposition: If $\langle f|A| h\rangle$ and $\langle f|B| h\rangle$ are defined then $\langle f| A+$ $+B|h\rangle$ is defined. If $\langle f|A| h\rangle,\left\langle f|A| h^{\prime}\right\rangle,\left\langle f^{\prime}|A| h\right\rangle$ and $\left\langle f^{\prime}|A| h^{\prime}\right\rangle$ are defined, then $\left\langle f+f^{\prime}|A| h+h^{\prime}\right\rangle$ is defined. The matrix element $\langle f|A| h\rangle$ is linear in $h$, antilinear in $f$ and linear in $A$. The complex conjugate of $\langle f|A| h\rangle$ is $\left\langle h\left|A^{*}\right| f\right\rangle$.

Proof: (a) Assume that $(\bar{J}(f) \times J(h)) \cap J(A)$ and $(\bar{J}(f) \times J(h)) \cap$ $\cap J(B)$ are not empty. Let $\left\{r, r^{\prime}\right\} \in(J(h) \times \bar{J}(f)) \cap J(A)$ and $\left\{q, q^{\prime}\right\} \in$ $\epsilon(J(h) \times \bar{J}(f)) \cap J(B)$. Let $p \leqq r, q$ be such that $E_{I p} H_{p}=E_{I r} H_{r} \cap$ $\cap E_{I q} H_{q}$ and $s^{\prime} \geqq r^{\prime}, q^{\prime}$ be such that $E_{I^{\prime} s^{\prime}} F_{s^{\prime}}=E_{I^{\prime} r^{\prime}} F_{r^{\prime}}+E_{I^{\prime} q^{\prime}} F_{q^{\prime}}$. Then $\left\{p, s^{\prime}\right\} \in J(h) \times \bar{J}(f)$ by the results of Section 3c. Furthermore, $\left\{p, s^{\prime}\right\}$ is a common successor, in $\left(I \times I^{\prime}\right)_{2}$, of $\left\{r, r^{\prime}\right\}$ and of $\left\{q, q^{\prime}\right\}$. Since $J(A)$ and $J(B)$ are final subsets of $\left(I \times I^{\prime}\right)_{2}$, it follows that $\left\{p, s^{\prime}\right\} \in$ $\in J(A) \cap \bar{J}(B) \leqq J(A+B)$. So $(J(h) \times \bar{J}(f)) \cap J(A+B)$ contains $\left\{p, s^{\prime}\right\}$ and is consequently not empty. This means that $\langle f|A+B| h\rangle$ is defined.

(b) Assume that the sets $(J(h) \times \bar{J}(f)) \cap J(A),\left(J(h) \times \bar{J}\left(f^{\prime}\right)\right) \cap J(A)$, $\left(J\left(h^{\prime}\right) \times \bar{J}(f)\right) \cap J(A)$ and $\left(J\left(h^{\prime}\right) \times \bar{J}\left(f^{\prime}\right)\right) \cap J(A)$ are all non-empty. 
Let $\left\{a, a^{\prime}\right\} \in(J(h) \times \bar{J}(f)) \cap J(A), \quad\left\{b, b^{\prime}\right\} \in\left(J(h) \times \bar{J}\left(f^{\prime}\right)\right) \cap J(A)$, $\left\{c, c^{\prime}\right\} \in\left(J\left(h^{\prime}\right) \times \bar{J}(f)\right) \cap J(A)$ and $\left\{d, d^{\prime}\right\} \in\left(J\left(h^{\prime}\right) \times \bar{J}\left(f^{\prime}\right)\right) \cap J(A)$. Let $r \leqq a, b$ be such that $E_{I r} H_{r}=E_{I a} H_{a} \cap E_{I b} H_{b}$. Then $r \in J(h)$. Similarly, let $q \leqq c, d$ be such that $E_{I q} H_{q}=E_{I c} H_{c} \cap E_{I d} H_{d}$. Then $q \in J\left(h^{\prime}\right)$. Let $r^{\prime} \geqq a^{\prime}, c^{\prime}$ be such that $E_{I r^{\prime}} F_{r^{\prime}}=E_{I^{\prime} a^{\prime}} F_{a^{\prime}}+E_{I^{\prime} c^{\prime}} F_{c^{\prime}}$. Then $r^{\prime} \in \bar{J}(f)$. Similarly, let $q^{\prime} \geqq b^{\prime}, d^{\prime}$ be such that $E_{I^{\prime} q^{\prime}} F_{q^{\prime}}=E_{I^{\prime} b^{\prime}} F_{b^{\prime}}+$ $+E_{I^{\prime} d^{\prime}} F_{d^{\prime}}$. Then $q^{\prime} \in \bar{J}\left(f^{\prime}\right)$. Furthermore, $\left\{r, r^{\prime}\right\} \geqq_{2}\left\{a, a^{\prime}\right\} ;\left\{r, q^{\prime}\right\} \geqq_{2}$ $\geqq_{2}\left\{b, b^{\prime}\right\} ;\left\{q, r^{\prime}\right\} \geqq_{2}\left\{c, c^{\prime}\right\}$ and $\left\{q, q^{\prime}\right\} \geqq_{2}\left\{d, d^{\prime}\right\}$. Since $J(A)$ is a final subset of $\left(I \times I^{\prime}\right)_{2}$, it follows that all four pairs $\left\{r, r^{\prime}\right\},\left\{r, q^{\prime}\right\},\left\{q, r^{\prime}\right\}$ and $\left\{q, q^{\prime}\right\}$ belong to $J(A)$. Consequently Proposition 4.4 applies:

If $s \geqq r, q$ is such that $E_{I s} H_{s}=E_{I r} H_{r}+E_{I q} H_{q}$, and if $p^{\prime} \leqq r^{\prime}, q^{\prime}$ is such that $E_{I^{\prime} p^{\prime}} F_{p^{\prime}}=E_{I^{\prime} r^{\prime}} F_{r^{\prime}} \cap E_{I^{\prime} q^{\prime}} F_{q^{\prime}}$, then $\left\{s, p^{\prime}\right\} \in J(A)$. Notice that $s \in J(h) \cap J\left(h^{\prime}\right) \subseteq J\left(h+h^{\prime}\right)$ since $J(h)$ and $J\left(h^{\prime}\right)$ are final subsets. Similarly $p^{\prime} \in \bar{J}(f) \cap \bar{J}\left(f^{\prime}\right) \subseteq \bar{J}\left(f+f^{\prime}\right)$.

Consequently $\left\{s, p^{\prime}\right\} \in\left(J\left(h+h^{\prime}\right) \times \bar{J}\left(f+f^{\prime}\right)\right) \cap J(A)$ which shows that $\left\langle f+f^{\prime}|A| h+h^{\prime}\right\rangle$ is defined.

(c) The assertions about linearity and complex conjugation can be easily verified.

This concludes the proof of Proposition 4.11.

\section{d) Product of operators}

Let $H_{I}=\left[H_{r} ; E_{s r} ; I\right], F_{I^{\prime}}=\left[F_{r^{\prime}} ; E_{s^{\prime} r^{\prime}} ; I^{\prime}\right]$ and $G_{I^{\prime \prime}}=\left[G_{r^{\prime \prime}} ; E_{s^{\prime \prime} r^{\prime \prime}}\right.$; $\left.I^{\prime \prime}\right]$ be nested Hilbert spaces. Let $A \in L\left(H_{I} ; F_{I^{\prime}}\right)$ and $B \in L\left(F_{I^{\prime}} ; G_{I^{\prime \prime}}\right)$.

Assume that $R(A) \cap D(B)$ is not empty (see Section 4 a for notations). Then

4.12 Proposition: The operator $E_{2}\left(I \times I^{\prime \prime} ; r, r^{\prime \prime}\right) B_{r^{\prime \prime} r^{\prime}} A_{r^{\prime} r} \in L\left(H_{I}\right.$; $\left.G_{I^{\prime \prime}}\right)$ is independent of the choice of $r^{\prime} \in R(A) \cap D(B),\left\{r, r^{\prime}\right\} \in J(A)$ and $\left\{r^{\prime}, r^{\prime \prime}\right\} \in J(B)$.

Proof: Let $r^{\prime} \in R(A) \cap D(B), \quad q^{\prime} \in R(A) \cap D(B), \quad\left\{r, r^{\prime}\right\} \in J(A)$, $\left\{q, q^{\prime}\right\} \in J(A),\left\{r^{\prime}, r^{\prime \prime}\right\} \in J(B)$ and $\left\{q^{\prime}, q^{\prime \prime}\right\} \in J(B)$. Let $s^{\prime} \geqq r^{\prime}, q^{\prime}$ be such that $E_{I^{\prime} s^{\prime}} F_{s^{\prime}}=E_{I^{\prime} r^{\prime}} F_{q^{\prime}}+E_{I^{\prime} q^{\prime}} F_{q^{\prime}}$. Then $\left\{s^{\prime}, r^{\prime \prime}\right\} \in J(B)$ and $\left\{s^{\prime}, q^{\prime \prime}\right\} \in$ $\in J(B)$ by Proposition 4.4. If $s^{\prime \prime} \geqq r^{\prime \prime}, q^{\prime \prime}$ and if $p \leqq r, q$, then

$$
\begin{gathered}
E_{s^{\prime \prime} r^{\prime \prime}} B_{r^{\prime \prime} r^{\prime}} A_{r^{\prime} r} E_{r p}=E_{s^{\prime \prime} r^{\prime \prime}} B_{r^{\prime \prime} s^{\prime}} E_{s^{\prime} r^{\prime}} A_{r^{\prime} r} E_{r p} \\
=E_{s^{\prime \prime} r^{\prime \prime}} B_{r^{\prime \prime} s^{\prime}} A_{s^{\prime} r} E_{r p}=B_{s^{\prime \prime} s^{\prime}} A_{s^{\prime} p} .
\end{gathered}
$$

Similarly $E_{s^{\prime \prime} q^{\prime \prime}} B_{q^{\prime \prime} q^{\prime}} A_{q^{\prime} q} E_{q p}=B_{s^{\prime \prime} s^{\prime}} A_{s^{\prime} p^{\prime}}$. So

$$
E_{s^{\prime \prime} r^{\prime \prime}} B_{r^{\prime \prime} r^{\prime}} A_{r^{\prime} r} E_{r p}=E_{s^{\prime \prime} q^{\prime \prime}} B_{q^{\prime \prime} q^{\prime}} A_{q^{\prime} q} E_{q D}
$$

which proves the proposition.

The operator defined by Proposition 4.12 will be denoted by $B A$ and called the product of $B$ and of $A$. 
So: The product $B A$ is defined if and only if the intersection of the final subset $R(A) \leqq I^{\prime}$ and of the initial subset $D(B) \leqq I^{\prime}$ is not empty.

4.13 Proposition: If $B A$ and $C A$ are defined, then $(B+C) A$ is defined. If $B A$ and $B C$ are defined then $B(A+C)$ is defined. The product $B A$ is linear in $B$ and in $A$.

Proof: (a) Assume that $R(A) \cap D(B)$ and $R(A) \cap D(C)$ are not empty. Let $r^{\prime} \in R(A) \cap D(B)$ and $q^{\prime} \in R(A) \cap D(C)$. Let $p^{\prime} \leqq r^{\prime}, q^{\prime}$ be such that $E_{I p^{\prime}} F_{p^{\prime}}=E_{I^{\prime} r^{\prime}} F_{r^{\prime}} \cap E_{I^{\prime} q^{\prime}} F_{q^{\prime}}$. Then $p^{\prime} \in R(A)$ by (4.13), by Theorem 3.9, and by the Corollary to Proposition 4.4. Furthermore $p^{\prime} \in D(B) \cap D(C)$ since $D(B)$ and $D(C)$ are initial subsets of $I^{\prime}$. So $p^{\prime} \in D(B+C) \geqq D(B) \cap D(C)$.

This proves that $R(A) \cap D(B+C)$ is not empty i. e. that $(B+C) A$ is defined.

(b) Assume that $R(A) \cap D(B)$ and $R(C) \cap D(B)$ are not empty. Let $r^{\prime} \in R(A) \cap D(B)$ and $q^{\prime} \in R(C) \cap D(B)$. Let $s^{\prime} \geqq r^{\prime}, q^{\prime}$ be such that $E_{I^{\prime} s^{\prime}} F_{s^{\prime}}=E_{I r^{\prime}} F_{r^{\prime}}+E_{I q^{\prime}} F_{q^{\prime}}$. Then $s^{\prime} \in D(B)$ by the corollary to Proposition 4.4. Furthermore $s^{\prime} \in R(C) \cap R(A) \leqq R(C+A)$ since $R(C)$ and $R(A)$ are final subsets of $I^{\prime}$. So $s^{\prime} \in R(C+A) \cap D(B)$ which proves that $B(A+C)$ is defined.

(c) The assertion about linearity is immediately verified. This concludes the proof of the proposition.

4.14 Proposition: Let $A \in L\left(H_{I} ; F_{I^{\prime}}\right)$ and $B \in L\left(F_{I^{\prime}} ; G_{I^{\prime \prime}}\right)$ be such that $B A$ is defined. Then $A^{*} B^{*}$ is defined and

$$
A^{*} B^{*}=(B A)^{*} \text {. }
$$

Proof: Let $\left\{r, r^{\prime}\right\} \in J\left(A^{*}\right)$ and $\left\{r^{\prime}, r^{\prime \prime}\right\} \in J(B)$. Then, by (4.12), $\left\{\bar{r}^{\prime \prime}, \bar{r}^{\prime}\right\} \in$ $\in J\left(B^{*}\right)$ and $\left\{\bar{r}^{\prime}, \bar{r}\right\} \in J(A)$. So $\bar{r}^{\prime} \in R\left(B^{*}\right) \cap D\left(A^{*}\right)$ which shows that $A^{*} B^{*}$ is defined. Furthermore, the representative of $A^{*} B^{*}$ between $G_{\bar{r}^{\prime \prime}}$ and $H_{\bar{r}}$ is

$$
\begin{aligned}
& \left(A^{*} B^{*}\right)_{\bar{r} \bar{r}^{\prime \prime}}=\left(A^{*}\right)_{\bar{r} \bar{r}^{\prime}}\left(B^{*}\right)_{\bar{r}^{\prime} \bar{r}^{\prime \prime}} \\
& =u_{\bar{r} r}\left(A_{r^{\prime} r}\right)_{r r^{\prime}}^{*} u_{r^{\prime} \bar{r}^{\prime}} u_{\bar{r}^{\prime} r^{\prime}}\left(B_{r^{\prime \prime} r^{\prime}}\right)_{r^{\prime} r^{\prime \prime}}^{*} u_{r^{\prime \prime} \bar{r}^{\prime \prime}} \\
& =u_{\bar{r} r}\left(B_{r^{\prime \prime} r^{\prime}} A_{r^{\prime} r}\right)_{r r^{\prime \prime}}^{*} u_{r^{\prime \prime} \bar{r}^{\prime \prime}}=u_{\bar{r} r}\left((B A)_{r^{\prime \prime} r}\right)_{r r^{\prime \prime}}^{*} u_{r^{\prime \prime} \bar{r}^{\prime \prime}} \\
& =\left((B A)^{*}\right)_{\bar{r} \bar{r}^{\prime \prime}}
\end{aligned}
$$

which proves the assertion.

\section{e) Linear functionals and their adjoints}

The set $C$ of complex numbers, being a one-dimensional Hilbert space, can be considered as a nested Hilbert space. If $H_{I}$ is an arbitrary nested Hilbert space, then one can study the spaces $L\left(C ; H_{I}\right)$ and $L\left(H_{I} ; C\right)$, defined as in Section 4 a. 
Consider first the space $L(C ; r)$ of linear maps from $C$ into the Hilbert space $H_{r}$. If $h_{r}$ is any vector in $H_{r}$, denote by $\left.\mid h_{r}\right)$ the map which associates, to every $\zeta \in C$, the vector $\left.\mid h_{r}\right) \zeta=\zeta h_{r} \in H_{r}$. Clearly $\left.\mid h_{r}\right) \in L(C ; r)$. Every element of $L(C ; r)$ can be written in the form $\left.\mid f_{r}\right)$, with a unique $f_{r} \in H_{r}$.

If $s \geqq r(r \in I)$, then the correspondence (4.1) becomes

$$
\left(h_{r}\right) \rightarrow E_{s r}\left(h_{r}\right) \text {. }
$$

In the present discussion, the set $I$ corresponds to the set $I^{\prime}$ and also to the set $\left(I \times I^{\prime}\right)_{2}$ of Section 4 a.

Notice that

$$
\left.\left.E_{s r} \mid h_{r}\right)=\mid E_{s r} h_{r}\right) .
$$

Indeed, for every $\zeta \in C$, one has $\left.E_{s r} \mid h_{r}\right) \zeta=E_{s r} \zeta h_{r}=\zeta E_{s r} h_{r}$ $\left.=\mid E_{s r} h_{r}\right) \zeta$.

Following Section $4 \mathrm{a}$, define $L\left(C ; H_{I}\right)$ as the algebraic inductive limit of the spaces $L(C ; r)$ with respect to the mappings (4.16). Denote by $E_{2}(I ; r)$ the natural embedding of $L(C ; r)$ into $L\left(C ; H_{I}\right)$.

4.15 Proposition: Let $h \in H_{I}$, and let $r, q$ be elements of $J(h)$. Then $\left.\left.E_{2}(I ; r) \mid h_{r}\right)=E_{2}(I ; q) \mid h_{q}\right)$. In other words: The element $\left.E_{2}(I ; r) \mid h_{r}\right)$ of $L\left(C ; H_{I}\right)$ does not depend on the choice of $r \in J(h)$.

Proof: If $h_{r}$ and $h_{q}$ are representatives of $h$, then there exists an $s \geqq r, q$ such that $E_{s r} h_{r}=E_{s q} h_{q}$. By (4.17), then, $\left.\left.E_{s r} \mid h_{r}\right)=E_{s q} \mid h_{q}\right)$ which proves the assertion.

For every $h \in H_{I}$, denote by $|h\rangle$ the element of $L\left(C ; H_{I}\right)$ defined by Proposition 4.15.

4.16 Proposition: The correspondence between $h$ and $|h\rangle$ is a linear bijection between $H_{I}$ and $L\left(C ; H_{I}\right)$.

Proof: (a) It is easy to verify that the correspondence $h \rightarrow|h\rangle$ is linear and injective.

(b) Let $A$ be any element of $L\left(C ; H_{I}\right)$ and let $A_{r c}$ be any one of its representatives. Then there exists a $h_{r} \in H_{r}$ such that $\left.A_{r c}=\mid h_{r}\right)$, since $A_{r c} \in L(C ; r)$. With the help of (4.17) one sees that $A=E_{I r} h_{r}$. This proves the proposition.

Consider now the mapping $\zeta \rightarrow|h\rangle \zeta$, defined as in Section $4 \mathrm{~b}$. It is given by

$$
|h\rangle \zeta=\zeta h \text {. }
$$

Indeed, if $\left.\mid h_{r}\right)$ is any representative of $|h\rangle$, then $\left.|h\rangle \zeta=E_{2}(I ; r) \mid h_{r}\right) \zeta$ $=\zeta E_{I r} h_{r}=\zeta h$.

Notice also that

$$
R(|h\rangle)=J(h) .
$$

Indeed, if $r \in J(h)$ and if $h_{r}$ is the representative of $h$ in $H_{r}$, then $\left.\mid h_{r}\right)$ is the representative of $|h\rangle$ in $L(C ; r)$. Conversely if $\left.\mid h_{r}\right)$ is a representative of $|h\rangle$ in $L(C ; r)$, then $h_{r}$ is a representative of $h$ in $H_{r}$. 
The set $D(|h\rangle)$ is trivial; it consists of a single element.

Denote by $\langle h|$ the adjoint of $|h\rangle$ (see Section $4 \mathrm{c}$ ). Then $\langle h|$ is an element of $L\left(H_{I} ; C\right)$. It follows from Theorem 4.9 that every element of $L\left(H_{I} ; C\right)$ can be written in the form $\langle h|$ in a unique way.

It follows from (4.13) that $D(\langle h|)=\bar{J}(h)$ so that the domain of the linear mapping $g \rightarrow\langle h| g$ is $H_{\tilde{J}(h)}$.

Let $r \in I$ and $h_{r} \in H_{r}$. Then the (Hilbert space) adjoint of the mapping $\left.\mid h_{r}\right)$ is the mapping $\left(h_{r} \mid\right.$ which associates, to every $g_{r} \in H_{r}$, the number $\left(h_{r}, g_{r}\right)$. From the definition 4.10 it follows that the representative of $\langle h|$ between $H_{\bar{r}}$ and $C$ is $\left(h_{r} \mid u_{r}\right.$.

So: If $g \in H_{\bar{J}(g)}$, then

$$
\langle h| g=\langle h \mid g\rangle .
$$

The scalar product $\langle h \mid g\rangle$ is defined if and only if $\langle h| g$ is defined.

These results can be put into a form which is reminiscent of the classical Riesz-Fréchet theorem on the representations of continuous linear functionals in a Hilbert space.

Let $D \leqq I$ be an initial subset which has the following property:

$\left(D_{1}\right)$ If $r \in D$ and $q \in D$ are arbitrary, then there exists an $s \geqq r, q$ such that $s \in D$ and that $E_{I s} H_{s}=E_{I r} H_{r}+E_{I q} H_{q}$.

It follows from $\left(\mathrm{D}_{1}\right)$ that $H_{D}=\bigcup_{r \in D} E_{I r} H_{r}$ is a vector subspace of $H_{I}$.

Define a regular linear functional in $H_{I}$ as a complex-valued linear mapping $l$ which

(i) Is defined on a vector subspace of the form $H_{D}$, where $D$ is an initial subset of $I$ satisfying the condition $\left(\mathrm{D}_{1}\right)$.

(ii) Is bounded in the following sense: If $r \in D$, then there exists a number $\gamma_{r}>0$ such that

$$
\left|l\left(E_{I r} h_{r}\right)\right| \leqq \gamma_{r}\left\|h_{r}\right\|
$$

for every $h_{r} \in H_{r}$. The dependence of $\gamma_{r}$ on $r \in D$ is arbitrary.

(iii) Is maximal in the following sense: There exists no linear functional $\tilde{l}$ satisfying (i), (ii) and such that $l$ is a proper restriction of $\tilde{l}$.

Then

4.17 Proposition: For every $h \in H_{I}$, the mapping

$$
g \rightarrow\langle h \mid g\rangle \quad\left(g \in H_{\bar{J}(h)}\right)
$$

is a regular linear functional.

Conversely, if $l$ is any regular linear functional in $H_{I}$, then there exists one and only one $h \in H_{I}$ such that $l$ is the mapping (4.22).

The proof consists in showing that regular linear functionals are precisely the ones given by $L\left(C ; H_{I}\right)$. It will be omitted.

Let $H_{I}$ and $F_{I^{\prime}}$ be nested Hilbert spaces. Let $h \in H_{I}$ and $f \in F_{I^{\prime}}$ be arbitrary. Then the product $|f\rangle\langle h| \in L\left(H_{I} ; F_{I^{\prime}}\right)$ is defined by the criterion of Section $4 \mathrm{~b}$. Indeed $R(\langle h|)$ and $D(|f\rangle)$ are the trivial one- 
element set that indexes $C$. If $g \in H_{\tilde{J}(h)}$, then $|f\rangle\langle h| g=\langle h \mid g\rangle f$. Notice that e. g. $D(|f\rangle\langle h|)$ may contain (properly) $D(\langle h|)=H_{\tilde{J}(h)}$. This is the case if $f=0$ and $J(h) \neq I$.

\section{Nested Hilbert space associated to an orthonormal basis}

We are given a separable Hilbert space $H_{0}$ and an orthonormal basis $\left\{h_{0}^{(k)}\right\}(k=1,2, \ldots)$ in $H_{0}$. It is shown below that there exists a nested Hilbert space around $H_{0}$ which is, in a certain sense, naturally associated to this basis. A suitable choice of $H_{0}$ and of the basis $\left\{h_{0}^{(k)}\right\}$ allows us to construct nested Hilbert spaces with desirable special properties. In order to have the results in a form ready for applications, we shall consider the case that the basis $\left\{h_{0}^{(k)}\right\}$ is given as an $n$-tuple sequence.

\section{a) The set $I^{(n)}$}

Throughout this part of the paper, $n$ will be a fixed integer such that $n \geqq 1$. Let

$$
r(k)=r\left(k_{1}, \ldots, k_{n}\right) \quad\left(k_{1}, k_{2}, \ldots, k_{n}=1,2, \ldots\right)
$$

be an $n$-tuple sequence of strictly positive numbers $(r(k)>0)$. In the set $I^{(n)}$ of all such sequences define an order relation by writing $r \geqq p$ if and only if $r(k) \geqq p(k)$ for every $k$. There exists in $I^{(n)}$ an orderreversing involution: If $r \in I^{(n)}$, then $\bar{r}$ is defined by $\bar{r}(k)=1 / r(k)$. Notice that the element $o$, defined by $o(k)=1$ for every $k$, satisfies $o=\bar{o}$. Furthermore, a common successor of $r \in I^{(n)}$ and of $q \in I^{(n)}$ is $\max (r(k)$, $q(k))$. So the set $I^{(n)}$ satisfies the conditions of Section $2 \mathrm{e}$.

\section{b) The Hilbert spaces $H_{r}$}

Let $H_{0}$ be a separable Hilbert space and

$$
\left.\left\{h_{0}^{(k)}\right\}=\left\{h_{0}^{\left(k_{1}\right.} \ldots k_{n}\right)\right\} \quad\left(k_{1}, \ldots, k_{n}=1,2,3, \ldots\right)
$$

an orthonormal basis of $H_{0}$. Denote by $V$ the subset of $H_{0}$ consisting of the finite linear combinations of the vectors $h_{0}^{(k)}$. Let $r$ be any element of $I^{(n)}$. Consider the prehilbert space obtained by defining the scalar product of $f_{0} \in V$ and of $g_{0} \in V$ as

$$
\sum_{k}\left(f_{0}, h_{0}^{(k)}\right) r^{-2}(k)\left(h_{0}^{(k)}, g_{0}\right) .
$$

(The sum contains only a finite number of non-zero terms.) Denote by $H_{r}$ the completion of this prehilbert space; it is a Hilbert space.

5.1 Proposition: If $s \geqq r\left(r \in I^{(n)}\right)$ then $H_{s} \geqq H_{r}$. The natural embedding of $H_{r}$ into $H_{s}$ is a nesting - to be denoted by $E_{s r}$ - which satisfies

$$
\left\|E_{s r}\right\| \leqq 1 \text {. }
$$


The family of nestings $E_{s r}\left(r \in I^{(n)}, s \geqq r\right)$ satisfies the conditions $\left(\operatorname{Ind}_{1}\right)$ and $\left(\operatorname{Ind}_{2}\right)$ of Section $2 \mathrm{f}$.

Proof: Let $r \in I^{(n)}$ be given. To every $f_{0} \in V$ associate the sequence $\left(h_{0}^{(k)}, f_{0}\right)$ of complex numbers. The closure (in $H_{r}$ ) of this correspondence is a unitary mapping from $H_{r}$ onto $l^{(2)}(\mu)$ where $\mu$ is the discrete measure given by $\mu_{k}=r^{-2}(k)$. The assertions then follow from Propositions 2.9 and 2.5. (Every unitary mapping is a nesting.)

\section{c) The nested Hilbert space $H_{I}^{(n)}$}

Let $H_{I}^{(n)}=\left[H_{r} ; E_{s r} ; I^{(n)}\right]$ be the algebraic inductive limit of the family $H_{r}$, considered in the preceding section, with respect to the nestings $E_{s r}$. The purpose of this section is to show that $H_{I}^{(n)}$ is a nested Hilbert space. Denote by $E_{I r}$ the natural embedding of $H_{r}$ into $H_{I}^{(n)}$. Consider in $H_{I}^{(n)}$ the vectors

$$
h^{(k)}=E_{I 0} h_{0}^{(k)} .
$$

Here $\left\{h_{0}^{(k)}\right\}$ is the orthonormal basis (5.1). For every $r \in I^{(n)}$, consider also the vectors

Notice that

$$
e^{(r ; k)}=r(k) h^{(k)} .
$$

and that

$$
J\left(h^{(n)}\right)=J\left(e^{(r ; k)}\right)=I^{(n)}
$$

$$
\left\|h_{r}^{(k)}\right\|=r^{-1}(k) .
$$

By (5.2), the representatives $e_{r}^{(r: k)}$ form an orthonormal basis in $H_{r}$ :

$$
\left(e_{r}^{(r: k)}, e_{r}^{(r: j)}\right)=\delta_{k j} .
$$

Here $\delta_{k j}=\delta_{k_{1} j_{1}} \ldots \delta_{k_{n} j_{n}}$.

It follows from (5.4) and (5.5) that, for $s \geqq r$,

$$
E_{s r} e_{r}^{(r ; k)}=\frac{r(k)}{s(k)} e_{s}^{(s ; k)} .
$$

Furthermore, by (5.7) and (5.8),

$$
\left(E_{s r}\right)_{r s}^{*} e_{s}^{(s ; k)}=\frac{r(k)}{s(k)} e_{r}^{(r ; k)} .
$$

For every $r \in I^{(n)}$, define $u_{\bar{r} r}$ by

$$
u_{\bar{r} r} e_{r}^{(r ; k)}=e_{\bar{r}}^{(\bar{r} ; k)},
$$

linear extension and closure. It is clear that $u_{\bar{r} r}$ is a unitary mapping from $H_{r}$ onto $H_{\bar{r}}$.

5.2 Proposition: The family $u_{\bar{r} r}$ satisfies the condition $\left(\mathrm{NH}_{2}\right)$ of Section $3 a$.

Proof: (a) $u_{\overline{0} 0}=1$ by (5.10), since $\bar{o}=o$. 
(b) Remember that $\bar{r}(k)=1 / r(k)$. Then, by (5.10), (5.8) and (5.9),

$$
\begin{aligned}
u_{r \bar{r}} E_{\bar{r} \bar{s}} u_{\bar{s} s} e_{s}^{(s ; k)} & =u_{r \bar{r}} E_{\bar{r} \bar{s}} e_{\bar{s}}^{(\bar{s} ; k)} \\
& =\frac{\bar{s}(k)}{\bar{r}(k)} u_{r \bar{r}} e_{\bar{r}}^{(\bar{r} ; k)}=\frac{r(k)}{s(k)} e_{r}^{(r ; k)}=\left(E_{s r}\right)_{r s}^{*} e_{s}^{(s ; k)} .
\end{aligned}
$$

Since this is true for every $k$, the assertion of the proposition follows.

Let $f \in H_{I}^{(n)}$, and $r \in J(f)$. Then, by (5.10) and (5.5)

$$
\begin{aligned}
\left(f_{r}, f_{r}\right) & =\sum_{k}\left|\left(f_{r}, e_{r}^{(r ; k)}\right)\right|^{2}=\sum_{k}\left|\left(u_{\bar{r} r} f_{r}, u_{\bar{r} r} e_{r}^{(r ; k)}\right)\right|^{2} \\
& =\sum_{k}\left|\left(u_{\bar{r} r} f_{r}, e_{\bar{r}}^{(\bar{r} ; k)}\right)\right|^{2}=\sum_{k} \bar{r}^{2}(k)\left|\left(u_{\bar{r} r} f_{r}, h_{\bar{r}}^{(k)}\right)\right|^{2} \\
& =\sum_{k} r^{-2}(k)\left|\left(u_{\bar{r} r} f_{r}, h_{\bar{r}}^{(k)}\right)\right|^{2} .
\end{aligned}
$$

Let $f \in H_{I}^{(n)}$ and $r \in J(f)$. Then the representative $f_{r}$ can be expanded with respect to the basis $\left\{e_{r}^{(r ; k)}\right\}$. This gives

$$
\begin{aligned}
f_{r} & =\sum_{k}\left(f_{r}, e_{r}^{(r ; k)}\right) e_{r}^{(r ; k)}=\sum_{k}\left(u_{\bar{r} r} f_{r}, u_{\bar{r} r} e_{r}^{(r ; k)}\right) e_{r}^{(r ; k)} \\
& =\sum_{k}\left(u_{\bar{r} r} f_{r}, e_{\bar{r}}^{(\bar{r} ; k)}\right) e_{r}^{(r ; k)}=\sum_{k}\left(u_{\bar{r} r} f_{r}, h_{\bar{r}}^{(k)}\right) h_{r}^{(k)}
\end{aligned}
$$

where the sums are all strongly convergent in $H_{r}$.

Let $f \in H_{I}^{(n)}$, and $r, q \in J(f)$. Let $a$ be any common predecessor of $r$ and of $q$. Then, with the help of (5.6) we obtain

$$
\begin{aligned}
\left(u_{\bar{r} r} f_{r}, h_{\bar{r}}\right) & =\left(u_{\bar{r} r} E_{r a} f_{a}, h_{\bar{r}}\right)=\left(f_{a},\left(E_{r a}\right)_{a r}^{*} u_{r \bar{r}} h_{\bar{r}}\right) \\
& =\left(f_{a}, u_{a \bar{a}} E_{\bar{a} \bar{r}} h_{\bar{r}}\right)=\left(f_{a}, u_{a \bar{a}} h_{\bar{a}}\right) .
\end{aligned}
$$

Similarly $\left(u_{\bar{q} q} f_{q}, h_{\bar{q}}\right)=\left(f_{a}, u_{a \bar{a}} h_{\bar{a}}\right)$, so that

$$
\left(u_{\bar{r} r} f_{r}, h_{\bar{r}}\right)=\left(u_{\bar{q} q} f_{q}, h_{\bar{q}}\right) .
$$

5.3 Proposition: Let $r$ and $q$ be any two elements of $I^{(n)}$. Define $p \in I^{(n)}$ by

$$
p(k)=\min (r(k), q(k)) .
$$

That is: For every $k, p(k)$ is the smaller of the two numbers $r(k), q(k)$. Then $p \leqq r, q$ and

$$
E_{I p} H_{p}=E_{I r} H_{r} \cap E_{I q} H_{q} .
$$

Proof: The only part of the statement that requires proof is $E_{I p} H_{p} \supseteqq$ $\supseteqq E_{I r} H_{r} \cap E_{I q} H_{q}$. We shall first prove the following assertion: Let $c(k)$ be an $n$-tuple sequence of numbers such that the series $\sum_{k} c(k) h_{r}^{(k)}$ and $\sum_{k} c(k) h_{q}^{(k)}$ are strongly convergent, in $H_{r}$ and $H_{q}$ respectively. Then the series $\sum_{k} c(k) h_{p}^{(k)}$ is strongly convergent in $H_{p}$. 
Indeed, denote by $\Sigma^{\prime}$ the summation over a finite subset. Then, by (5.7) and the orthogonality of the $h_{r}^{(k)}$ (resp. the $\left.h_{q}^{(k)}\right)$, we have

$$
\begin{aligned}
\left\|\Sigma^{\prime} c(k) h_{r}^{(k)}\right\|^{2} & =\sum_{k}^{\prime}|c(k)|^{2} r^{-2}(k) \\
\left\|\Sigma^{\prime} c(k) h_{q}^{(k)}\right\|^{2} & =\sum_{k}^{\prime}|c(k)|^{2} q^{-2}(k) \\
\left\|\Sigma^{\prime} c(k) h_{p}^{(k)}\right\|^{2} & =\sum_{k}^{\prime}|c(k)|^{2} p^{-2}(k) \leqq \sum_{k}^{\prime}|c(k)|^{2}\left(r^{-2}(k)+q^{-2}(k)\right) \\
& =\left\|\sum_{k}^{\prime} c(k) h_{r}^{(k)}\right\|^{2}+\left\|\sum_{k}^{\prime} c(k) h_{q}^{(k)}\right\|^{2} .
\end{aligned}
$$

So: If the partial sums of $\sum_{k} c(k) h_{r}^{(k)}$ and of $\sum_{k} c(k) h_{q}^{(k)}$ are Cauchy sequences, then the partial sums of $\sum_{k} c(k) h_{p}^{(k)}$ are also Cauchy sequences. Since $H_{p}$ is complete, the series $\sum_{k} c(k) h_{p}^{(k)}$ is strongly convergent.

Now let $f \in E_{I r} H_{r} \cap E_{I q} H_{q}$. Write $c(k)=\left(u_{\bar{r} r} f_{r}, h_{r}^{(k)}\right)=\left(u_{\bar{q}_{Q}} f_{q}, h_{q}^{(k)}\right)$ [see (5.13)]. Then, by (5.12), we have the strongly convergent expansion

$$
\begin{aligned}
& f_{r}=\sum_{k} c(k) h_{r}^{(k)} \\
& f_{q}=\sum_{k} c(k) h_{q}^{(k)} .
\end{aligned}
$$
It follows that the series $\sum_{k} c(k) h_{p}^{(k)}$ is strongly convergent in $H_{p}$. Denote
its sum by $f_{p}^{\prime}$. Then

$$
E_{r p} f_{p}^{\prime}=\sum_{k} c(k) E_{r p} h_{p}^{(k)}=\sum_{k} c(k) h_{r}^{(k)}=f_{r}
$$

which shows that $f_{p}^{\prime}$ should be denoted by $f_{p}$ and that it is the representative of $f$ in $H_{p}$. So $f \in E_{I p} H_{p}$ which proves the proposition.

Acknowledgements. It is a pleasure to acknowledge the hospitality of the Courant Institute of Mathematical Sciences during the academic years 1963-1964 and 1964-1965 when most of this work was completed.

\section{References}

[1] Schwartz, L.: J. d'Analyse Mathématique (Jerusalem), XIII, 115 (1964).

[2] Grossmann, A.: J. Math. Phys. 6, 54 (1965).

[3] Gel'fand, I. M., and N. Ya Vilenkin: Generalized Functions, Volume IV. Moscow: State Publishing House of Mathematical and Physical Literature 1961.

[4] RIEsz, F., and B. Sz-NAGY: Lecons d'analyse fonctionnelle. Budapest: Akademiai Kiado 1952.

[5] DUNFord, N., and J. T. Schwartz: Linear operators I. New York: Interscience 1958.

[6] Bourbaki, N.: Eléments de mathématique; Théorie des ensembles, Fascicule de résultats. Paris: Hermann 1963.

[7] - Eléments de mathématique; Algèbre linéaire. Paris: Hermann 1962.

[8] Halmos, P. R.: Measure theory. Toronto, New York, London: Von Nostrand 1950. 